CALT-TH-2020-051

\title{
A violation of global symmetries from replica wormholes and the fate of black hole remnants
}

\author{
Po-Shen Hsin ${ }^{1}$, Luca V. Iliesiu ${ }^{2}$ and Zhenbin Yang ${ }^{2}$ \\ ${ }^{1}$ Walter Burke Institute for Theoretical Physics, California Institute of Technology, \\ Pasadena, CA 91125, USA \\ ${ }^{2}$ Stanford Institute for Theoretical Physics, Stanford University, Stanford, CA 94305, USA
}

\begin{abstract}
We show that the presence of replica wormholes in the Euclidean path integral of gravity leads to a non-perturbative violation of charge conservation for any global symmetry present in the low-energy description of quantum gravity. Explicitly, we compute the scattering probability between different charged states in several low-dimensional models of quantum gravity and find a non-vanishing answer. This suggests that the set of all charged states is typically over-complete, which has drastic consequences for the fate of black hole remnants that could carry a global symmetry charge. In the holographic context, we argue that the presence of such a symmetry in the effective description of the bulk should appear on the boundary as an emergent global symmetry after ensemble averaging.
\end{abstract}




\section{Contents}

1 Introduction $\quad 2$

2 Review of previous arguments

2.1 Hawking's original argument . . . . . . . . . . . . . . 5

2.2 The remnant argument . . . . . . . . . . . . . . . . 6

2.3 The Harlow-Ooguri and Harlow-Shaghoulian argument . . . . . . . . . . . 7

3 Global symmetry violation from replica wormhole 9

3.1 General argument . . . . . . . . . . . . . . . . . 9

3.2 A few examples . . . . . . . . . . . . . . . . 12

3.2.1 JT gravity coupled to matter . . . . . . . . . . . . 13

3.2.2 Gravity coupled to a pure gauge theory . . . . . . . . . . . [16

3.3 Differences between global and gauge symmetries . . . . . . . . . . 18

3.4 How the global symmetry $G$ can arise and the factorization problem . . . . 19

4 Charged state reconstructions $\quad 22$

4.1 General argument . . . . . . . . . . . . . . . . . 22

4.2 Planar Resummations in JT gravity . . . . . . . . . . . . . . . 26

5 The fate of remnants $\quad 29$

6 Conclusion and comparison to past arguments 32

A Squared one- and two-point function

B State reconstruction in the canonical ensemble 37 


\section{Introduction}

A longstanding question in quantum gravity is whether exact global symmetries can be present [1, 2, 3, 4, 5, 6, 7, 8, 9]. When such global symmetries are present, a problem appears when forming black holes from particles that carry an overall global symmetry charge. A semiclassical analysis of evaporation suggests that the Hawking radiation emitted by such black holes is thermal [10, 11, 12]. Consequently, the global symmetry charge of the black hole cannot significantly change through the evaporation process. If the black hole evaporates completely and the resulting state of Hawking radiation is a singlet under the global symmetry (as suggested by the semiclassical analysis), then the evaporation process violates the conservation of the global symmetry charge, a central tenet of global symmetries in quantum field theory. Alternatively, the black hole might not evaporate completely but rather decay to some remnant state that retains the global symmetry charge of the black hole. Because in the process of evaporation, a large number of indistinguishable small black holes can be formed [6], each with a different global symmetry charge 1 such states have a much larger degeneracy than what is allowed by the "central dogma" [13], which states that from the perspective of an outside observer black holes describe quantum systems with $S_{\mathrm{BH}}=$ (horizon area) $/\left(4 G_{N}\right)$ degrees of freedom.

The primary goal of this paper is to show that if a global symmetry is present in the effective description in any theory of quantum gravity, ${ }^{2}$ then the presence of (replica) wormholes in the Euclidean path integral of the theory leads to a non-perturbative violation of this global symmetry. ${ }^{3}$

To probe the violation of charge conservation, we compute the scattering probability between states with different global symmetry charges. On a black hole background, we find that this probability is non-zero. In contrast to our common intuition from quantum field theory, this implies that states with different global symmetry charges in quantum gravity are non-orthogonal and could form an over-complete basis. Consequently, to understand whether small black holes or remnants indeed disobey the "central dogma" discussed above, we determine the minimal basis of charged states that spans the space of states for such

\footnotetext{
${ }^{1}$ For now, we assume that the global symmetry is continuous or, if discrete, has a large number of irreducible unitary representations.

${ }^{2}$ Here, we can consider the case where the global symmetry is present in the effective description up to arbitrarily large energy scales.

${ }^{3}$ There have been several past papers making the connection between the existence of wormhole solutions and the violation of global symmetries in quantum gravity [3, 14]. However, the wormholes discussed in this paper are different in nature, i.e. they appear because we are considering the gravitational path integral in the presence of multiple boundaries.
} 
objects. In contrast to the previous analysis, which suggests that the dimension of this basis is equal to the number of unitary irreducible representations of the global symmetry group [6]. 4 we find that the dimension is always given by $\sim e^{S_{\mathrm{BH}}}$ after considering the contribution of connected geometries in the gravitational path integral. The result is now consistent with the "central dogma" and suggests that, in principle, black holes that carry a global symmetry charge can fully evaporate.

It is perhaps not surprising that the contribution of (replica) wormholes to the gravitational path integral drastically alters the conclusions of the semiclassical analysis for black hole evaporation. Recently, by considering the contribution of replica wormholes, [15, 16, 13] reproduced the correct behavior of the Page curve at late times, further providing a detailed map of how modes trapped in the interior of the black hole are encoded in the Hawking radiation at late times. The analysis presented in this paper is closely related to these developments, as we explain how global symmetry charges trapped inside the black hole horizon can "escape" through replica wormholes analogous to those considered in [15, 16].

Even though the conservation of global symmetry charge is violated due to wormhole contributions, one might ponder the origin of this symmetry in the effective gravitational theory, in the context of holography. For bulk theories with multiple boundaries, the contribution of wormholes to the gravitational path integral leads to the widely discussed factorization puzzle [17]. To obtain boundary observables consistent with this lack of factorization in the bulk, we can consider a boundary system given by an ensemble average of theories [17]. In this context, we conjecture the following relation between the bulk and the boundary:

- If each theory in the ensemble average on the boundary has some global symmetry $G$, then the effective theory in the bulk should have a gauge symmetry whose gauge group is also given by $G$. This is the standard case in AdS/CFT [18].

- If the ensemble average on the boundary gives rise to some emergent global symmetry $G$ (which is not a symmetry of individual Hamiltonians in the ensemble), then the effective gravitational theory in the bulk should have the same global symmetry $G$. On the boundary, global symmetry charge conservation is violated when considering the average of several replicas of the ensemble, while in the bulk the violation occurs because of replica wormholes. This means that if we couple this boundary symmetry to a background gauge field, the replicated system will not be invariant under the most general gauge transformations. A concrete example to have in mind for a boundary theory exhibiting such features is the Sachdev-Ye-Kitaev (SYK) model [19, 20, 21, 22]; the model has an emergent $O(N)$ symmetry after ensemble averaging but has no such symmetry in individual instances of the ensemble.

\footnotetext{
${ }^{4}$ Of course, in the case of a continuous symmetry group, this number is infinite.
} 
This improvement of the holographic dictionary provides a new perspective on the problem of factorization. If we want to restore the factorization in the bulk with modifications of the Lagrangian of the theory or by finding some UV completion, this requires an explicit breaking of all bulk global symmetries in order to be consistent with the wormhole calculation.

The remainder of this paper is organized as follows. In section 2, we review several known arguments that suggest the absence of global symmetries in quantum gravity. In section 3 , we present the general setup for our calculation and compute the scattering amplitude between states of different charges in several toy models of gravity. Furthermore, we emphasize the difference between global and gauge symmetries in the gravitational path integral, explaining why the latter does not exhibit a violation of charge conservation, while the former does. We also discuss how global and gauge symmetries can arise in holographic theories with ensemble-averaged dual boundary theories. In section 4, we show that any states in the black hole interior can be reconstructed by an $e^{S_{\mathrm{BH}}}$ number of states. In Jackiw-Teitelboim (JT) gravity [23, 24, we do an exact planar resummation to find the reconstruction map, and explicitly find the complete basis of states. In section 5 we relate our findings to the problem of remnants and we speculate about their ultimate fate. Finally, in section 6 we summarize the main points of our paper and discuss their relation to past arguments against global symmetries in quantum gravity.

Note added: During the development of this paper, 25] appeared, and also discussed the violation of global symmetries in quantum gravity from a different perspective, also using replica wormholes.

\section{Review of previous arguments}

Before expanding on our arguments regarding the violation of global symmetries due to replica wormholes, it is instructive to first review several arguments about the absence of global symmetry in quantum gravity. We can summarize most of these arguments through figure 1, that shows the Penrose diagram of an evaporating black hole. As we will discuss later in the paper, each one of the arguments reviewed below can be refined by including the contribution of connected geometries to the gravitational path integral. 


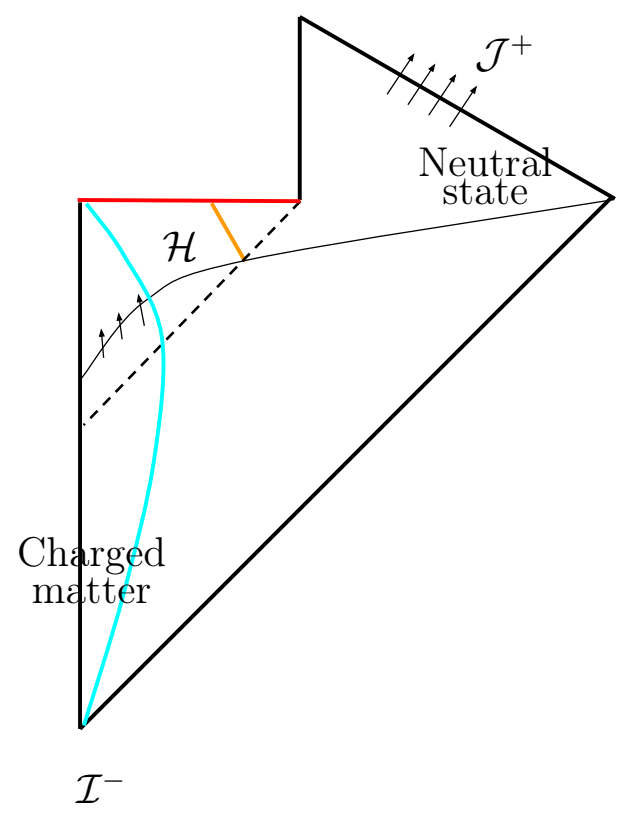

Figure 1: The Penrose Diagram of an evaporation black hole. At $\mathcal{I}^{-}$, we collide a large amount of particles, forming a representation $R$ under the global symmetry $G$, to create a black hole. The Hawking radiation at $\mathcal{J}^{+}$is thermal and independent of $R$ [10, 11]. The original matter is stored in the interior region $\mathcal{H}$, which causes a problem when the log of dimension of the representation $R$ exceeds the Bekenstein-Hawking entropy.

\subsection{Hawking's original argument}

Considering a quantum field theory with a continuous global symmetry coupled to gravity, we can create a black hole by colliding a shell of matter field carrying an overall non-zero global charge. In the absence of gauge fields for the symmetry (i.e. the symmetry is not gauged), the macroscopic structure of the black hole is insensitive to the global symmetry charge due to the no-hair theorem [26, 27, 28, 29]. This means that the Hawking radiation will be the same as the case of an ordinary Schwarzchild black hole. In the leading order approximation, only the lightest particles are produced in the evaporation, and they do not carry any of the original global symmetry charges and at the end of the evaporation process, assuming there are no remnants that carry the global symmetry charge, we have an almost thermal distribution of radiation. This means that the black hole evaporation process violates the charge conservation of the symmetry, and therefore global symmetry must be violated in quantum gravity [12]. The argument can be formulated quantitatively 
as a scattering process, by defining a dollar matrix that represents the transition amplitude between the initial and final density matrix: [12]

$$
\$_{m m^{\prime} ; n n^{\prime}} \rho_{n n^{\prime}}^{\text {in }}=\rho_{m m^{\prime}}^{\text {out }}
$$

which for a unitary process can be factorized as a product of S-matrix elements:

$$
\$_{m m^{\prime} ; n n^{\prime}}=S_{m n} S_{m^{\prime} n^{\prime}}^{*}
$$

For a single non-unitary evolution, the dollar matrix cannot be factorized. Then, the breaking of a global symmetry $G$ means that the dollar matrix does not commute with the symmetry transformation:

$$
\$_{m m^{\prime} ; n n^{\prime}} G_{n l} G_{n^{\prime} l^{\prime}}^{*} \neq G_{m n} G_{m^{\prime} n^{\prime}}^{*} \$_{n n^{\prime} ; l l^{\prime}}
$$

The above argument assumes that the black hole fully evaporates and no remnant is present. This, of course might be incorrect since the semiclassical computation of the rate of Hawking radiation can fail once the black hole mass becomes sufficiently small, say $M=X M_{p l}$ for some number $X$. As we will review below, the absence of global symmetries in quantum gravity can be motivated even when assuming the presence of remnants.

\subsection{The remnant argument}

While Hawking' original argument is physically intuitive, it relies on the assumption that nothing dramatic happens at the end point of the evaporation process where the semiclassical calculation may break down. The validity of Hawking's calculation can be estimated from the change of the black hole mass due to radiating one thermal quanta. For example, the temperature of a mass $M$ Schwarzchild black hole is of order $1 /\left(G_{N} M\right)$, which means that the semiclassical picture breaks down when $M \sim M_{p l}$.

Banks and Seiberg, on the other hand, provide an additional argument against global symmetries [6]. Just like in the setup described above, they imagine the initial matter forms a representation $R$ of a large dimension under some non-abelian group $G$. Under the black hole evaporation, the initial matter will remain in the interior region $\mathcal{H}$ of the black hole. The black hole can evaporate down to a mass $M=X M_{p l}$, where $X M_{p l}$ is the energy scale at which semiclassical thermodynamics breaks down and the result might be a long lived remnant. At this energy scale the entropy on its lightsheet (the non-expanding lightcone associated to its horizon) will be order $\sim X$ and could be smaller than log of the dimension of the representation $R$. Then the entropy of the interior modes exceeds the area of the almost evaporated black hole

$$
\frac{A}{4 G_{N}}=\pi X^{2}<\log \operatorname{dim} R<S_{\text {interior }} .
$$


The covariant entropy bound [30] states that the matter entropy on a lightsheet is bounded by the change of the transverse area: $S_{\text {lightsheet }} \leq \frac{\Delta A}{4 G_{N}}$. Assuming that the matter entropy on the lightsheet bounds the matter entropy in the black hole interior $S_{\text {lightsheet }} \sim S_{\text {interior }}$ (we will revisit this point in the end of this section), this leads to a contradiction.

The above argument can be improved to include the cases even if the group $G$ is abelian or it does not have representations with large dimensions. One instead considers forming several black holes with several representations $R \in \mathcal{R}$, for some large set of unitary irreducible representations $\mathcal{R}$. As explained before, since Hawking radiation is thermal, we can assume that the black holes maintain their representations $R$ throughout their evaporation process. When all black holes reach the mass $M=X M_{p l}$, we thus obtain a number $\sum_{R \in \mathcal{R}} \operatorname{dim} R$ of remnant states that are indistinguishable. Once again, if $\pi X^{2}<\log \sum_{R \in \mathcal{R}} \operatorname{dim} R$, making the same set of assumptions as above, the existence of such objects is inconsistent with the covariant entropy bound. Consequently, this stronger version of the above argument rules out the existence of any global symmetry with Lie group $G$ or any finite global symmetry $G$ with large enough unitary representations.

As previously hinted, the above arguments require several technical assumptions. The main technical assumption is that the entropy on the lightsheet can be related to the entropy on some space-like Cauchy slice stretching through the interior of the black hole/remnant. This relation is unclear when the lightsheet (drawn in orange in figure 1) intersects the singularity of the black hole (the red line) [31]. Thus, it is unclear whether the covariant entropy bound actually applies to the matter entropy on the whole interior slice of the black hole (or remnant) [5 Rather, it should be the "central dogma" [13] described in the introduction that bounds the number of states inside the black hole interior, at least seeing from outside. Furthermore, the argument that the entropy of the black hole exceeds the dimension of certain representations can be made only at late-times when the black hole has almost fully evaporated. It would therefore be interesting to understand whether recently discussed effects coming from the contribution of replica wormholes (which completely alter late-time observables, such as the entanglement entropy of the Hawking radiation [16, 15] or correlators of matter fields [34]), affect the conclusions of [6]. We will explicitly address this in section 4 and 5 .

\subsection{The Harlow-Ooguri and Harlow-Shaghoulian argument}

The holographic principle, or the AdS/CFT correspondence, provides well-defined quantum gravity theories from their boundary dual descriptions. In such context, Harlow and Ooguri

\footnotetext{
${ }^{5}$ There have been numerous other arguments against the existence of remnants [32, 33, however they require different technical assumptions which we do not address in this paper.
} 
construct a new argument against global symmetries in gravity using the idea of entanglement wedge reconstruction [7, 8]. Recently, Harlow and Shaghoulian [9] extended this argument to more general evaporating black holes using the recent development of the Page curve [15, 16, 35, 36, 37]. They imagine a setup in which the spacetime of an evaporating black hole is separated into two regions $S$ and $R$ : $S$ contains the black hole and is understood as some kind of boundary description, while $R$ stands for a "reservoir" absorbing the Hawking radiations and is understood as the exterior region where gravity effects are nonessential. The Page curve describes the entropy of the Hawking radiations in $R$. The entropy of the radiations first grows maximally due to the thermalization between $S$ and $R$ and is bounded by the Bekenstein-Hawking entropy of the black hole system, that means it should decrease after the Page time when it saturates the black hole entropy. In the gravity picture, such a transition corresponds to a phase transition of two Quantum Extremal Surfaces (QES) [38]: one is the empty surface and the other is close to the black hole horizon. After the Page time, the nontrivial QES will dominate and enclose a large portion of the black hole interior called the island, which belongs to the entanglement wedge of $R$ but not $S$. On the other hand, if one considers a smaller portion of $R$ when the naive thermal entropy of the system does not exceed the black hole entropy, there will be no Page transition and therefore no island. Based on this, Harlow and Shaghoulian argue that the unitary transformation generated by the global symmetry group can be split into products of unitary transformations on $S$ and small portions $R_{i}$ of $R\left(\cup_{i} R_{i}=R\right)$ :

$$
U(g)=U(g, S) \prod_{i} U\left(g, R_{i}\right) U_{\text {edge }}
$$

where $U_{\text {edge }}$ is only supported on the edges of $R_{i}$. After the Page time, the island region is not contained in any of the entanglement wedge of $S$ and $R_{i}$ 's. Such a global symmetry cannot act on any simple operators in the island, and therefore the global symmetry cannot exist.

Given that the island formula has recently been "derived" from the contribution of replica wormholes to the gravitational path integral [15, 16], it would be informative to understand why the global symmetry cannot exist without relying on the existence of islands. Rather, in the next sections we will directly address what effects Euclidean wormholes have on global symmetries present in quantum gravity. 


\section{Global symmetry violation from replica wormhole}

\subsection{General argument}

In this section, we will provide a new argument about the nonexistence of global symmetry in quantum gravity. We will argue that even if the Lagrangian of the theory preserves global symmetry, global symmetry charge conservation is violated in quantum gravity due to the existence of replica wormholes. More precisely, the replica wormhole will predict a nonzero transition probability between states with different symmetry global charges.

In order to understand the contribution of such replica wormholes, we should first list the necessary assumptions for computing observables in a gravitational theory. Throughout this paper we will be interested in preparing states in some gravitational theory, with metric $g_{\mu \nu}$, coupled to a matter field $\Phi$, such that the Lagrangian $\mathcal{L}\left(\Phi, g_{\mu \nu}\right)$ of the theory is invariant under some global transformation $G$. We can prepare such a state by using the Euclidean path integral, with Dirichlet boundary conditions for the metric (i.e. the Hartle-Hawking state $|H H\rangle$ ), which means that boundary operator insertions can be specified in a diffeomorphism invariant way. Thus, for some operator $\mathcal{O}(\tau)$ formed from the matter field $\Phi$, we can define some state $|\psi\rangle$ as,

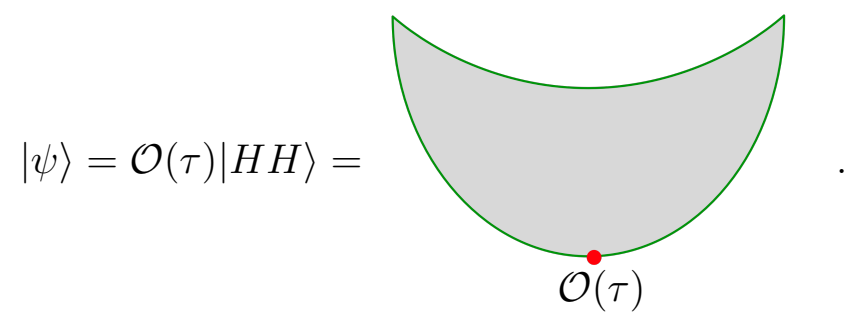

Matter excitations of the field $\Phi$ can be classified into representations of $G$. For example, consider two operators $\mathcal{O}_{1}, \mathcal{O}_{2}$ transforming under representation $R_{1}$ and $R_{2}$ of global symmetry $G$, which, when acting on the Hartle-Hawking state, create two states $\left|R_{1}\right\rangle$ and $\left|R_{2}\right\rangle$ with representation $R_{1}$ and $R_{2}$. A nonzero transition amplitude between these two states $\left\langle R_{2} \mid R_{1}\right\rangle=\left\langle\mathcal{O}_{2}^{\dagger} \mathcal{O}_{1}\right\rangle$ would imply that the global symmetry $G$ is broken, given that $\bar{R}_{1} \otimes R_{2}$ does not contain the singlet. The physical observable constructed from the transition amplitude between the two states is the scattering probability $\left|\left\langle R_{2} \mid R_{1}\right\rangle\right|^{2}$. For simplicity, we will for now consider the case in which there is no Lorentzian evolution between the in- and outstates and when the global symmetry $G$ is never spontaneously broken ${ }^{6}$ Without Lorentzian evolution, the scattering probability yields the squared norm of the inner-product between

\footnotetext{
${ }^{6}$ Even if $G$ is spontaneously broken we can always set the VEVs of charged fields to vanish by choosing appropriate boundary conditions. We will emphasize this through an example in section 3.2 .2 .
} 
the two states which, due to the charge conservation, would simply vanish in quantum field theory. This will not be the case when coupling $\Phi$ to gravity.

In terms of the gravitational path integral, the scattering probability can be written as

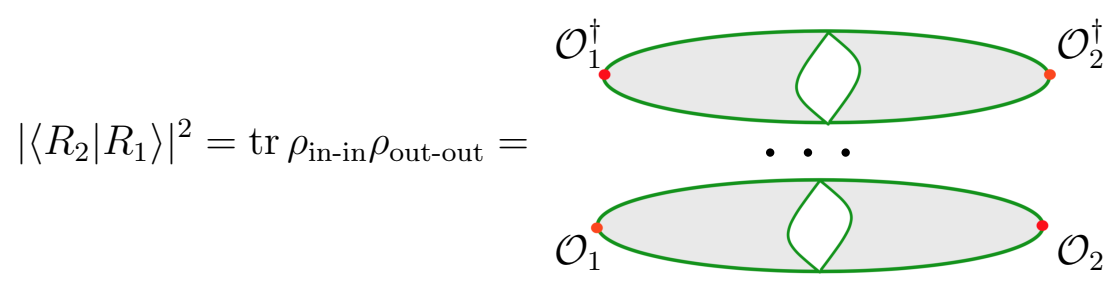

where we rewrite the this probability in terms of two density matrices $\rho_{\text {in-in }}=\left|R_{1}\right\rangle\left\langle R_{1}\right|$ and $\rho_{\text {out-out }}=\left|R_{2}\right\rangle\left\langle R_{2}\right|$ to (i) emphasize that just like when computing the Renyi entropies in a gravitational theory [15, 16], we need to consider several (for the scattering probability, only two) replicas of the gravitationally prepared density matrices, and (ii) to directly understand the relation of this probability to the $\$$-matrix considered in section 2.1 .

Next, we assume that between the two replicas in $(3.2)$, there is no restriction on the Euclidean gravitational path integral which would disallow connected geometries:7 Thus, the inner-product between the states $\left|R_{1}\right\rangle$ and $\left|R_{2}\right\rangle$ is given by

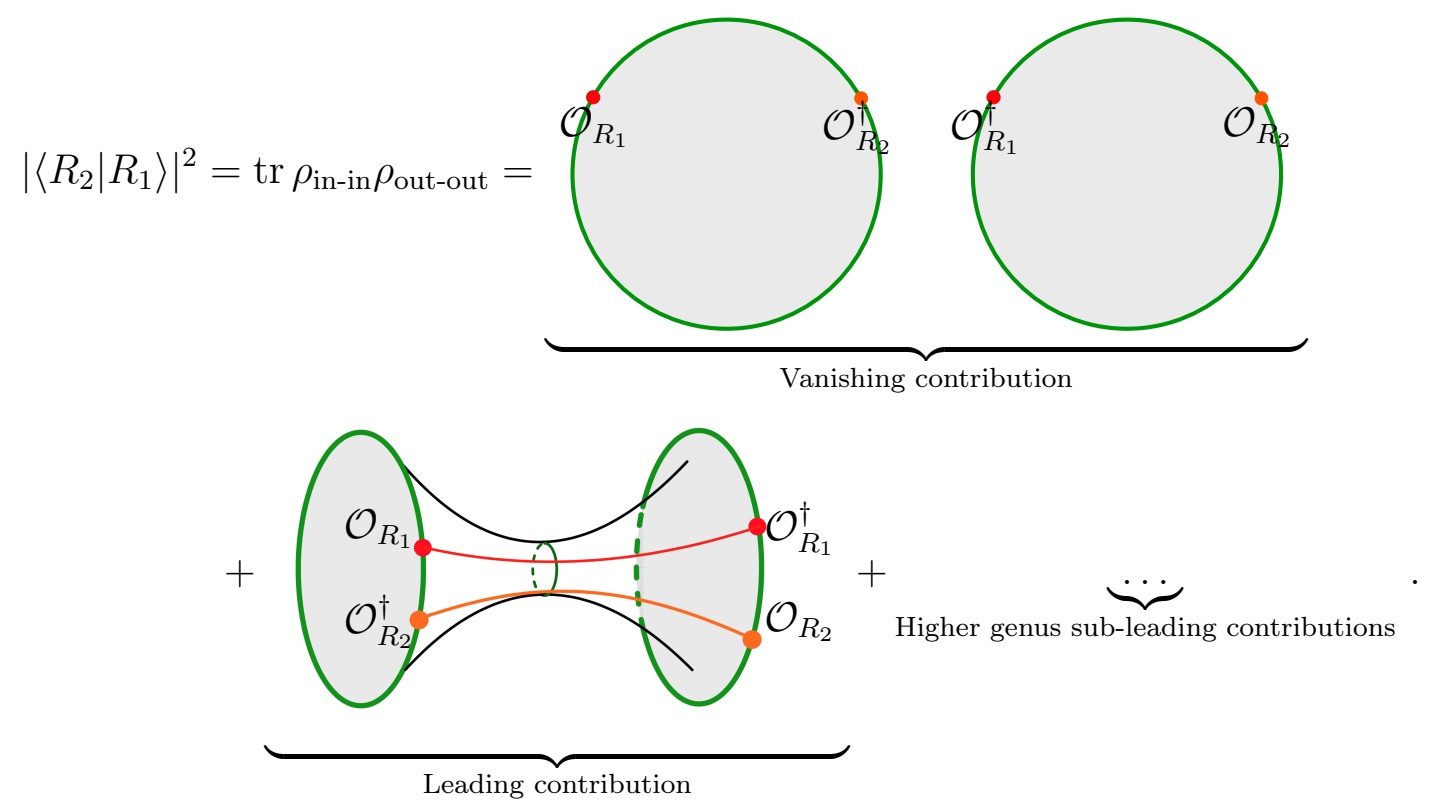

The leading disconnected geometries are given in the first line and their contribution is an integral over the correlators $\left\langle\mathcal{O}_{1}^{\dagger} \mathcal{O}_{2}\right\rangle$ and $\left\langle\mathcal{O}_{1} \mathcal{O}_{2}^{\dagger}\right\rangle$ on two disconnected fluctuating geometries.

${ }^{7}$ While we do not yet know of a reason whether to include (or exclude) connected replica geometries in a UV completion of gravity, from the path integral perspective, there is no way to impose that we only sum over connected geometries when using a local measure for the metric $g_{\mu \nu}$. 
However, since we have assumed that $\bar{R}_{1} \times R_{2}$ contains no singlets, the correlators $\left\langle\mathcal{O}_{1}^{\dagger} \mathcal{O}_{2}\right\rangle$ and $\left\langle\mathcal{O}_{1} \mathcal{O}_{2}^{\dagger}\right\rangle$ vanish on all backgrounds due to charge conservation, when assuming that the global symmetry $G$ is not spontaneously broken. The second line in 3.3 yields the contribution of the replica wormhole (i.e. the connected geometry) and its contribution is given by the correlator $\left\langle\mathcal{O}_{1}^{\dagger} \mathcal{O}_{2} \mathcal{O}_{1} \mathcal{O}_{2}^{\dagger}\right\rangle$ evaluated on a sum over fluctuating connected geometries. This correlator is generically non-vanishing on any geometry since singlets are always present in the tensor products $\bar{R}_{1} \times R_{1}$, and $\bar{R}_{2} \times R_{2}$ (following from the definition of the complex conjugate irreducible representation). The connected geometry yields a non-zero contribution and is especially trustworthy, even in a theory whose UV completion is unknown, when the replica wormhole geometry (in the presence of operator insertions) is a saddle point of the gravitational path integral. We will explicitly compute the value of this correlator in the next subsections in two simple toy-models: in section 3.2.1, in JT gravity coupled to a massive scalar field theory with a $U(1)$ global symmetry, where we will show that the wormhole is indeed a saddle for the black hole geometry, and, in section 3.2.2, in a simpler two-dimensional topological theory of gravity, coupled to a $\mathbb{Z}_{k}$ BF theory. The only other contributions to the inner-product are given by sub-leading, typically higher topology, geometries which are non-perturbatively suppressed either because they capture the contribution of sub-leading saddles or, as is the case for JT gravity [39], because they are exponentially suppressed by $e^{-2 \text { (BH entropy)g }}$ where $g$ is the genus of the connected manifold.

To obtain the scattering probability, we need to analytically continue the geometries above, to have a period of Lorentzian evolution between the in- and out-states. Such an analytic continuation is schematically given by,

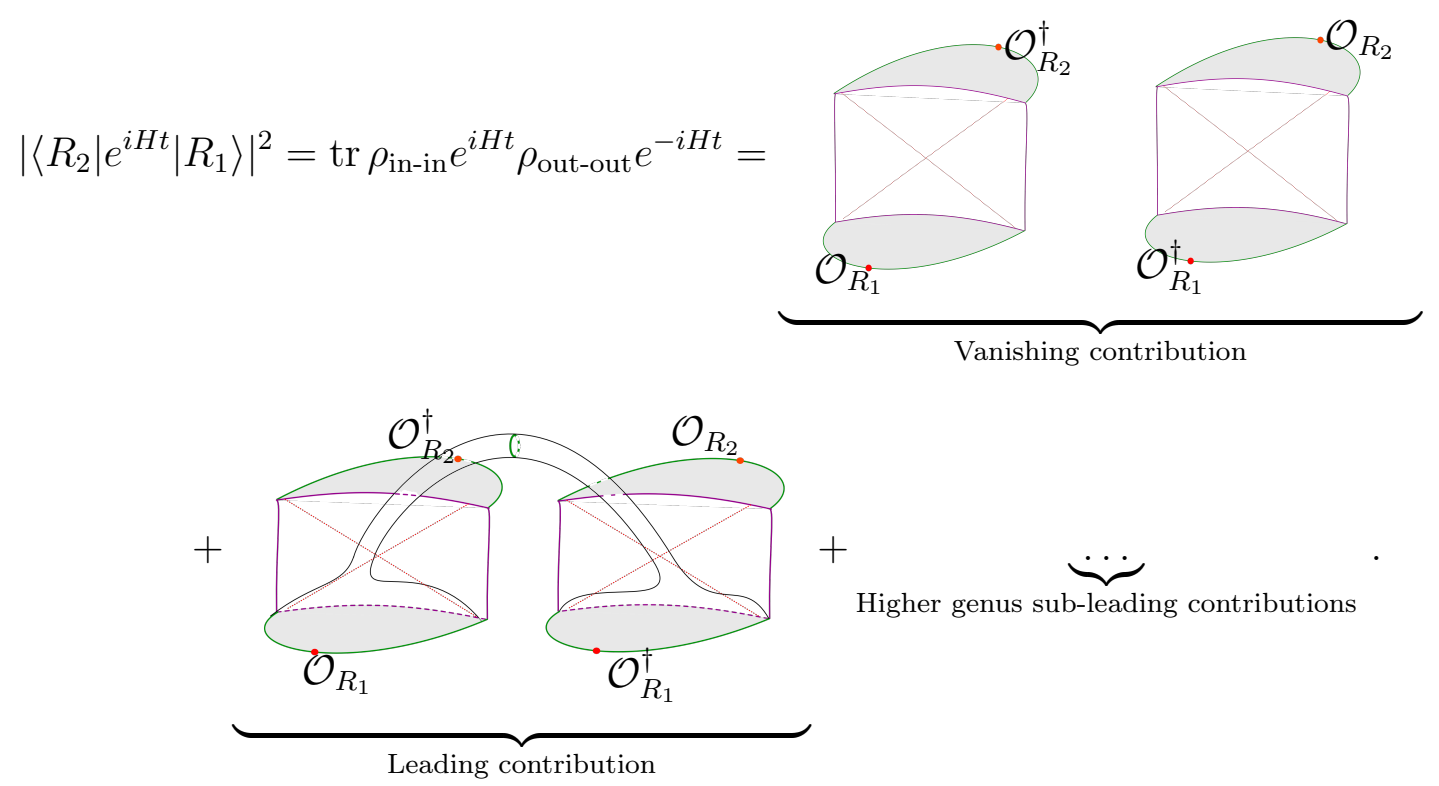

The first line, again shows the contribution of the vanishing disconnected geometries on the 
black hole background, whose horizon is shown by the red dotted lines and where periods of Lorentzian evolution are symbolized by green curves and periods of Lorentzian evolution are symbolized by purple boundary curves. The Lorentzian replica wormhole contribution is captured on the second line of (3.4), where it is unimportant the order in which connect the Euclidean patches. Notice that again, since the scattering probability is given by a simple analytic continuation of the inner-product in (3.3), although the wormhole is an instanton contribution to the gravitational path integral and is $e^{-\frac{1}{G_{N}}}$ suppressed, it dominates over the original geometry which gives a vanishing answer. Therefore we conclude that the transition probability, just like the inner-product norm, does not vanish and the charge conservation of the global symmetry $G$ is violated in quantum gravity, even when there is no explicit breaking of the symmetry in the Lagrangian. We can in principle consider more general density matrices $\rho_{\text {in-in }}$ and $\rho_{\text {out-out }}$ and the comments regarding the contributions of replica wormholes will still follow as long as the QFT path integral on some generic wormhole geometry is non-vanishing.

Finally, if the bulk theory has a boundary dual, where we have to consider an ensemble average of theories due to the wormholes, the nonzero transition probability between charged states in the bulk means that the corresponding inner-product between states on the boundary is some nonzero random number. After taking the ensemble average of a single copy of such a system, this inner-product vanishes, while if taking two copies of the system, needed to compute the absolute value of the inner-product, we find a non-vanishing answer. As previously described, this implies that the the system develops a global symmetry only after taking the ensemble average and we will discuss examples of such occurrences in section 3.4. This provides us a new aspect of the issue of factorization: if we want to restore the factorization in the bulk with a modifications of the Lagrangian of the theory, this inevitably requires an explicit breaking of all the global symmetries to be consistent with the wormhole calculation. We will discuss this point further in section 3.4 .

\subsection{A few examples}

In this section, we will provide a few examples that support our argument. The main examples are based on two dimensional gravitational theories where the wormhole configurations are best understood. However, we except the argument can extend to higher dimensions, where wormhole geometries can also be constructed [17]. 


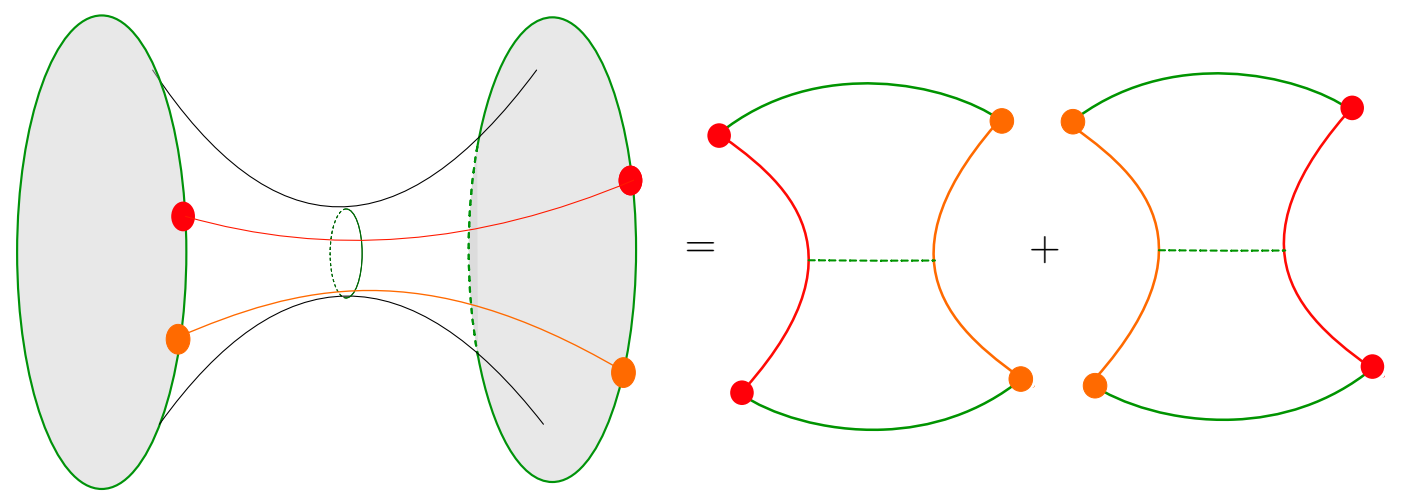

Figure 2: A decomposition of the Euclidean wormhole seen in the second line of (3.3) into patches on the Poincaré disk.

\subsubsection{JT gravity coupled to matter}

Our first example is JT gravity [24, 23] coupled with bulk matter field which has a global symmetry $G$. Such a theory is not UV complete due to the contribution of higher topology geometries to the path integral [39], so, in order to estimate the effect of such geometries, our strategy will be to look for semiclassical gravitational saddles. For concreteness and simplicity, we can take the matter field to be a complex scalar $\Phi=\varphi$ which carries some charge $q$ under a $U(1)$ subgroup of $G$. Consider two charge states $|q\rangle$ and $|\bar{q}\rangle$ prepared by insertion of $\varphi$ and $\varphi^{*}$ on a thermofield double state with inverse temperature $\beta$ :

$$
|q\rangle_{\beta}=\sum_{n} e^{-\frac{\beta}{4} H_{L}-\frac{\beta}{4} H_{R}} \varphi_{L}|n\rangle_{L}|n\rangle_{R}, \quad|\bar{q}\rangle_{\beta}=\sum_{n} e^{-\frac{\beta}{4} H_{L}-\frac{\beta}{4} H_{R}} \varphi_{L}^{*}|n\rangle_{L}|n\rangle_{R},
$$

prepared as in 3.1).

The inner-product between these two states is equal to the two-point function

$$
\langle\varphi(\beta / 2) \varphi(0)\rangle_{\beta}=\operatorname{Tr}\left(e^{-\frac{\beta}{2} H} \varphi e^{-\frac{\beta}{2} H} \varphi\right)
$$

on a single boundary. In JT gravity, such a correlation function is given by a summation over all hyperbolic geometries ending on the single boundary, including the disk topology and adding handles on it. As we discussed in the section above, the two-point function $\langle\varphi(\beta / 2) \varphi(0)\rangle_{\beta}$ on such geometries is identically zero, due to the existence of the U(1) symmetry.

Let's now consider the absolute value squared of the inner-product, $|\langle\bar{q} \mid q\rangle|^{2}$ as in $(3.3)$. In JT gravity, such a two boundary correlator is given by summation over all smooth hyperbolic 
geometries ending on the two boundaries, including factorized geometries and non-factorized geometries. The correlator on the factorized geometry is zero, since there will be two independent $\mathrm{U}(1)$ global symmetry acting on the two boundaries and for each boundary the insertion of the operators $\left(\varphi \varphi\right.$ and $\left.\varphi^{*} \varphi^{*}\right)$ is not $U(1)$ invariant. On the other hand, the correlator on the non-factorized geometry can generically be nonzero, since now there will be only a unique global $U(1)$ symmetry acting on these two boundaries, and the whole quantity is invariant under such global transformations.

Our next step is then to construct such a semiclassical wormhole geometry. The leading wormhole geometry that connects between these two asymptotic boundaries is the doubletrumpet geometry (the second line in $(3.3)$ ). On such geometries, there exists non-vanishing bulk propagators connecting $\varphi$ to $\varphi^{*}$ on the two sides.8 In JT gravity, such a configuration can be evaluated directly by cutting the double trumpet along the geodescis connecting $\phi$ and $\phi^{*}$, along which the particle propagate semi-classically when their masses is sufficiently large (see figure 2). Denoting the geodesic distance along the two geodesics as $\ell_{1,2}$, the propagator can be approximated as $e^{-m \ell_{1,2}}$. The gravitational path integral on the rectangular region with two asymptotic boundaries $\beta_{L, R}$ and two geodesic lengths $\ell_{1,2}$ is then given by [41, 42, 43, 44, 45, 34, 40]:

$$
\left\langle\ell_{1}\left|e^{-\beta_{L} H_{L}-\beta_{R} H_{R}}\right| \ell_{2}\right\rangle=\int d E \rho(E) e^{-\left(\beta_{L}+\beta_{R}\right) E}\left\langle\ell_{1} \mid E\right\rangle\left\langle E \mid \ell_{2}\right\rangle,
$$

where $\rho(E)=\frac{1}{2 \pi^{2}} \sinh (2 \pi \sqrt{2 E})$ is the density of states and $\langle\ell \mid E\rangle=4 K_{2 i \sqrt{2 E}}\left(4 e^{-\ell / 2}\right)$ [42, 43]. We will come back to this later, but, for the moment, let's assume the matter partition function can be ignored. Then the whole path integral can be obtained by gluing these two rectangular regions with the propagator:

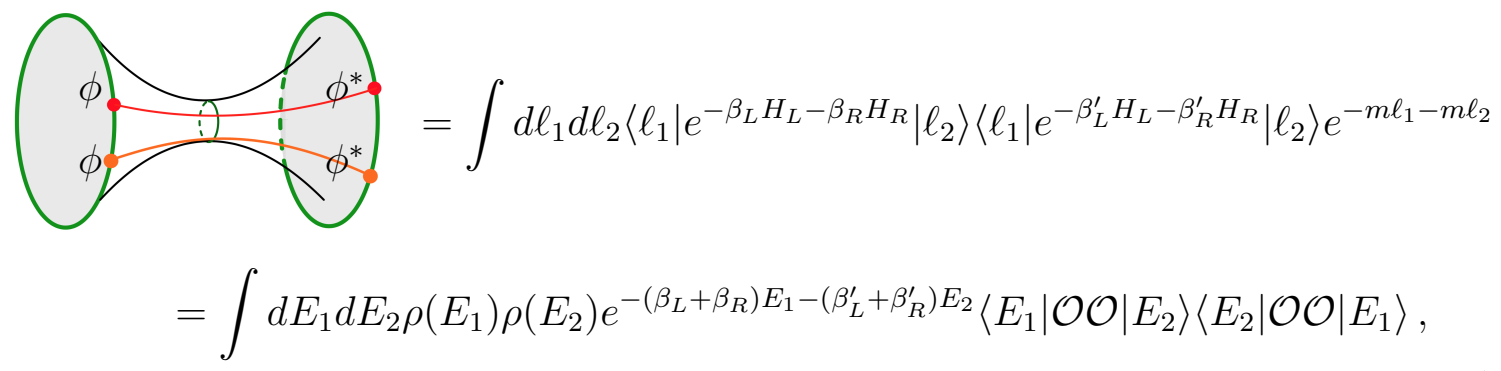

where $\left\langle E|\mathcal{O O}| E^{\prime}\right\rangle$ is the two point function in energy basis on a disk [41, 42, 43, 44, 45]:

$$
\left\langle E|\mathcal{O} \mathcal{O}| E^{\prime}\right\rangle=\frac{\Gamma\left(m \pm i\left(\sqrt{2 E} \pm \sqrt{2 E^{\prime}}\right)\right)}{2^{2 m+1} \Gamma(2 m)},
$$

\footnotetext{
${ }^{8}$ Such a quantity has recently been considered by Stanford [40] and is further reviewed in appendix A using the exact quantization method of JT gravity.
} 
where the \pm sign above means we need to take product of all the four gamma functions coming from different choice of the \pm signs.

In the symmetric configuration where $\beta_{L}+\beta_{R}=\beta_{L}^{\prime}+\beta_{R}^{\prime}=\beta, E_{1,2}$ has the same saddle point $E$. The action of $E$ contains two pieces: the gravitational action contributes the usual thermal action, $S(E)-\beta E$, and the propagator contributes an action of order $m \log E-S(E)$ coming from the asymptotic expansion of the gamma functions.

Together, this leads to a semiclassical saddle of the energy:

$$
E_{\text {saddle }}=\frac{m}{\beta} \text {. }
$$

Now, let's examine our assumption of ignoring the matter partition function. In order to justify that, we need the size of the wormhole, $b$ (i.e. the geodesic length across the wormhole), to be large. In appendix A, this size has be estimated directly using the cross ratio of the four corners of the rectangular region. As a result, when we do a Lorentzian time evolution $\beta_{L, R} \rightarrow \beta_{L, R} \pm i T$ and $\beta_{L, R}^{\prime} \rightarrow \beta_{L, R}^{\prime} \mp i T$, the size of the wormhole grows linearly with time:

$$
b \sim 2 \sqrt{2 E_{\text {saddle }}} T .
$$

Therefore, as long as $T$ is large enough, we can ignore the contribution of the matter partition function. The wormhole contribution is then given by:

$$
\left|\langle\bar{q} \mid q\rangle_{\beta}\right|^{2} \sim \frac{1}{\left|\langle q \mid q\rangle_{\beta}\right|^{2}}\left(\frac{8 m}{\beta}\right)^{2 m} e^{-2 m} \frac{\Gamma(m)^{4}}{2^{4 m} \Gamma(2 m)^{2}} \sim\left(\frac{\beta m}{2 \pi^{2}}\right)^{2 m} e^{-2 S_{0}-\frac{4 \pi^{2}}{\beta}-2 m},
$$

where we assume $m$ is large. $\langle q \mid q\rangle$ is a normalization factor, and is given by the disk two point function:

$$
\langle q \mid q\rangle_{\beta} \sim \frac{\Gamma(m)^{2}}{2^{2 m} \Gamma(2 m)}\left(\frac{4 \pi}{\beta}\right)^{2 m} e^{\frac{S_{0}+2 \pi^{2}}{\beta}} .
$$

Finally, let's remark on the situation when one of the operators whose scattering probability we want to compute is the identity operators. Once again, the leading wormhole is the double-trumpet geometry and the same cutting and the gluing rule explained above can be used to obtain the expectation value if we ignore the contribution of the matter partition function [34]. The result is similar as the two point function case:

$$
\left|\langle\varphi\rangle_{\beta}\right|^{2}=\int d E \rho(E) e^{-\left(\beta_{L}+\beta_{R}\right) E}\langle E|\mathcal{O O}| E\rangle .
$$

The saddle point discussion is almost identical with the two point function case, so we will not repeat that analysis here. Again, to justify the assumption of neglecting the matter partition function, one needs to do the same analytic continuation of $\beta_{L, R} \rightarrow \beta_{L, R} \pm i T$. The only difference between this case and the two point function case is that the total boundary time evolution in this case is mostly Lorentzian, just like the situation of the spectrum form factor. 


\subsubsection{Gravity coupled to a pure gauge theory}

Our second example is $2 d$ gravity coupled to a BF theory [46, 47], the $2 d$ gravity could either be pure topological gravity, JT gravity [39, 48, 49] or its extensions [50, 51]. The point of considering such a theory is two-fold:

- The first is that the theory is UV complete so we should not worry about the matter partition function divergence coming from wormholes with small size $b$.

- The second is that such a theory has a spontaneous breaking of its zero-form symmetry (whose origin we review below) and we will be able to show that the general analysis in section 3.1 is applicable even in such a case.

The action of the theory is:

$$
S_{\text {grav }}[g]+\frac{i k}{2 \pi} \phi d a+S_{\text {boundary }}(g, \Phi, a)
$$

where $a$ is a $U(1)$ gauge field, and $\phi$ is a scalar with periodicity $2 \pi$, which enforces the gauge field $a$ to be flat by the equation of motion. $k$ is quantized to be an integer in order for the action to be well-defined for $2 \pi$-periodic scalar $\phi$ : under the "gauge transformation" $\phi \rightarrow \phi+2 \pi$, the action changes by $i k \int d a$. For it to be a multiple of $2 \pi i, k$ needs to an integer by the Dirac quantization condition $\oint d a \in 2 \pi \mathbb{Z}$.

Let us focus on the BF theory, which is a topological field theory that does not couple to the gravity. It describes a $\mathbb{Z}_{k}$ gauge theory [52, 6, 53. The theory has the following operators

$$
U=e^{i \phi}, \quad V=e^{i \oint a}, \quad U^{k}=V^{k}=1, \quad U V U^{-1}=e^{2 \pi i / k} V,
$$

where the last relation means these operators have non-trivial braiding given by $k$ th root of unity, as can be seen from the Aharonov-Bohm phase. Thus if we ignore gravity, the global symmetries of the theory are:

- $\mathbb{Z}_{k}$ zero-form symmetry: the symmetry is generated by $V$, and $U$ carries the unit charge. The symmetry acts as $\phi \rightarrow \phi+\lambda_{0}$ where $\lambda_{0}$ is a multiple of $2 \pi / k$. This leaves the action invariant using the Dirac quantization condition $\oint d a \in \mathbb{Z} q^{9}$

- $\mathbb{Z}_{k}$ one-form symmetry: the one-form symmetry [54] is generated by $U$, and $V$ carries the unit charge. The one-form symmetry acts as $a \rightarrow a+\lambda_{1}$ for one-form $\lambda_{1}$ with $\mathbb{Z}_{k}$ holonomy.

\footnotetext{
${ }^{9}$ In contrast, the gauge transform for the gauge group is $a \rightarrow a+d \lambda^{\prime}$ that leaves $\phi$ invariant.
} 
The above symmetries should not be confused with the $\mathbb{Z}_{k}$ gauge symmetry.

Within the topological field theory in the absence of dynamical gravity, these symmetries are spontaneously broken: for instance, on spatial circle there are $k$ vacua labelled by $e^{i \phi}$ which is a $k$ th root of unity. On the other hand, when the operator $U$ or $V$ is inserted in homologically non-trivial cycle, the expectation value in the topological field theory vanishes [54].

Now, let us consider the theory on a disk $D$ with the Dirichlet boundary condition for the gauge field $a$. The disk partition function for such a theory is given by,

$$
Z_{B F}[a]=\sum_{q=1}^{k} \chi_{q}\left(e^{i \oint_{\partial D} a}\right)
$$

where each term in the sum corresponds to one of the $k$ vacua and $\chi_{q}\left(e^{i \oint_{\partial D} a}\right)=e^{i q \oint_{\partial D}{ }^{a}}$ is the $\mathbb{Z}_{k}$ character with $q \in 1, \ldots k$.

Setting $a=0$, we want to prove that the one-point function of $U$ inserted at the boundary of the disk vanishes,

$$
\langle U\rangle=0
$$

To see this, we can modify the action by the insertion of $U=e^{i \phi}$ at some point $p$ :

$$
\phi(p)+\frac{k}{2 \pi} \int \phi d a=\int\left(\frac{k}{2 \pi} \phi d a+\phi \delta(p)^{\perp}\right)
$$

where $\delta(p)^{\perp}$ is a delta function that restricts the integral to $p$. Then the equation of $\phi$ implies

$$
d a=-\frac{2 \pi}{k} \delta(p)^{\perp} .
$$

If $p$ formed the boundary end points of a curve $\gamma$, then the equation can be solved with $a=-\frac{2 \pi}{k} \delta(\gamma)^{\perp}$. On the other hand, here we only have $U$ inserted at a single point and no such $\gamma$ exists, and the equation cannot be satisfied. Thus, with the appropriate boundary condition $(a=0)$ the correlation function equals zero despite the fact that the $\mathbb{Z}_{k}$ zero-form symmetry is spontaneously broken.

Instead, if we consider the one-point function squared $|\langle U\rangle|^{2}$, there are two leading contributions: two disjoint disks with $U$ and $U^{\dagger}$ inserted at their boundaries, and cylinder that connects the two disks ( similar to the second line of $(3.3)$ with $\mathcal{O}_{2}=\mathbb{1}$ ). The first contribution vanishes similar to the disk one-point function. The second contribution is however nonzero: when normalized in pure BF theory, the correlation function in the topological field theory is $1 / k$. More precisely, if the purely gravitational amplitude decreases with the genus and equals $e^{-S_{\text {disk }}}$ for the disk and $e^{-S_{\text {cylinder }}}$ for the cylinder, then the leading contribution 
to the one-point function squared is

$$
|\langle U\rangle|^{2}=\frac{e^{2 S_{\text {disk }}-S_{\text {cylinder }}}}{k}+\cdots,
$$

where the $\cdots$ represents sub-leading corrections. To see this, note the equation 3.20 can now be solved with $a=-\frac{2 \pi}{k} \delta(\ell)^{\perp}$ with $\ell$ a curve connecting the two insertions of $U$ on the top and the bottom of the cylinder. Then the contribution to the correlation function is nonzero. Thus, just like in the previous analysis, the $\mathbb{Z}_{k}$ symmetry is explicitly violated.

\subsection{Differences between global and gauge symmetries}

In this section, we will discuss how our argument is affected if the global symmetry is gauged. As previously mentioned, in the holographic context, having a gauge symmetry instead of a global symmetry in the bulk is a common occurrence [18].

By gauging a global symmetry $G$, we modify the Lagrangian by typically adding a gauge field, so that it is now invariant under local transformations of $G$. In the presence of boundaries, we can consider gauge transformations that do not vanish at the boundary but preserve the boundary conditions for the gauge field. In such a case, such a gauge symmetry acts on charged fields which can be placed on the boundary as a global symmetry. This means that in the presence of multiple boundaries, after gauging a global symmetry $G$ we find multiple global symmetries each acting separately on their own boundaries. Going back to our wormhole argument, this means that in order to have a non-vanishing result of the scattering probability, the operator insertions on each boundary need to be invariant under the global transformation, i.e. the gauge transformations that preserve the boundary conditions for the gauge field. That is, if the global symmetry is gauged, the transition probability between two different charge states is zero, and is therefore not broken by wormholes. Thus, in the example discussed in (3.2.1), if we gauge the $U(1)$ symmetry, under which complex scalar $\varphi$ has charge $q$, then $|\langle\bar{q} \mid q\rangle|^{2}=\langle\varphi \varphi\rangle\left\langle\varphi^{*} \varphi^{*}\right\rangle=0$ since the operators inserted on each boundary do not form a gauge singlet, regardless of whether we demand gauge transformations to vanish at each boundary ${ }^{10}$

For this $U(1)$ gauge theory, an alternative perspective can be obtained from Gauss's law. In such a case, the total charge going though the wormhole geometry needs to vanish due to the equation of motion: $d * F=j$, where $F$ is the field strength and $j$ is the current. Integrating this equation over the throat of the wormhole, which is a closed manifold, we automatically get the constraint $\oint j=0$.

\footnotetext{
${ }^{10}$ The correlators evaluated here are different than those for the non-local operator, $\varphi e^{\int{ }^{A}} \varphi^{*}$ which includes a Wilson line stretching between the ends of the wormhole. Rather, here we only consider insertions of $\varphi$ on the boundary.
} 
As we shall explain shortly, in a theory with a holographic dual, where the boundary theory is given by an ensemble average, a bulk gauge symmetry means that the symmetry is preserved in each realization of the ensemble. Two symmetries whose "gauging" has been extensively discussed in JT gravity [55], and are also present in individual instances of SYK models, are the fermion parity symmetry $(-1)^{F}$ or the time-reversal symmetry $\mathcal{T}$ of the boundary dual. We will discuss the role of these symmetries as well as bulk global symmetries in the following subsection.

\subsection{How the global symmetry $G$ can arise and the factorization problem}

While the previous discussions focused on the gravitational theory, we need to explain how the global symmetry $G$ can arise in the bulk, in a holographic theory with a boundary dual. Since we are considering the contribution of connected geometries for which the bulk partition function does not factorize, we will consider the dual to be given by an ensemble average of boundary theories. If the global symmetry $G$ is present in the bulk, then the same global symmetry $G$ should also be present on the boundary. There are two logical possibilities for the boundary global symmetry $G$ :

(i) $G$ is a global symmetry for each theory that is part of the ensemble.

(ii) $G$ arises only after ensemble averaging and is not a symmetry of each member of the ensemble.

We first analyze the case (i) ${ }^{11}$ Assuming that $G$ is not spontaneously broken in any of the members of the ensemble, then $\left|\left\langle\mathcal{O}_{R}\right\rangle\right|^{2}=:\left\langle\mathcal{O}_{R}\right\rangle\left\langle\mathcal{O}_{R}^{\dagger}\right\rangle:=0\left(\mathcal{O}_{R}\right.$ is some operator charged under the global symmetry $G$ and : $\cdots$ : indicate the point where we consider the ensemble average). This computation disagrees with the computation in section 3 of correlators of operators charged under $G$ in the gravitational theory. Therefore, we conclude that such a boundary symmetry cannot correspond to a bulk global symmetry and rather corresponds to a bulk gauge symmetry [48, 49] which is unaffected by the contribution of the replica wormholes (see 3.3 . .

For case (ii), after ensemble averaging, : $\left\langle\mathcal{O}_{R}\right\rangle:=0$; however, there is no reason for the expectation value : $\left|\left\langle\mathcal{O}_{R}\right\rangle\right|^{2}:=:\left\langle\mathcal{O}_{R}\right\rangle\left\langle\mathcal{O}_{R}^{\dagger}\right\rangle$ : to vanish once considering the ensemble average. We emphasize this point by considering example of the SYK model with $N$ Majorana fermions which, after ensemble averaging, has an emergent $O(N)$ symmetry. Such

\footnotetext{
${ }^{11}$ This case is also discussed when coupling JT gravity to a $2 \mathrm{~d}$ gauge theory as in [48, 49].
} 
a symmetry acts on the fermionic fields as $\psi_{i} \rightarrow U_{i j} \psi_{j}$ and on the random coupling as $J_{i_{1} \ldots i_{k}} \rightarrow J_{i_{1} \ldots i_{k}}\left(U^{-1}\right)_{i_{1} j_{1}} \ldots\left(U^{-1}\right)_{i_{1} j_{1}}$ and leaves the path integral for a single SYK copy invariant:

$$
Z_{S Y K} \sim \int d J_{i_{1} \ldots i_{q}} \int D \psi_{i} e^{-\int d \tau\left[\sum_{i=1}^{N} \psi_{i} \partial_{\tau} \psi_{i}-(i)^{\frac{q}{2}} J_{i_{1} \ldots i_{q}} \psi_{i_{1}} \ldots \psi_{i_{q}}\right]} .
$$

where $J_{i_{1} \ldots i_{q}}$ is the random coupling that is drawn from a Gaussian distribution with variance, $\left\langle j_{i_{1} \ldots i_{q}}^{2}\right\rangle=J^{2}(q-1) ! / N^{q-1}$. Importantly, since the emergent $O(N)$ symmetry requires that we transform the coupling $J_{i_{1} \ldots i_{k}}$, the measure for the integral which averages over this coupling is invariant under this $O(N)$ transformations.

We can now consider a charged operator which transforms under $O(N)$ but is a singlet under the discrete symmetries (such as the fermion parity $(-1)^{F}$ or the time reversal symmetry $\mathcal{T}$ ) that are present in each individual ensemble (we will discuss the case in which the operator is also charged under such discrete symmetries shortly). We can take such an operator to be $\mathcal{O}_{A}=i \psi_{[i} \psi_{j]}$ which transforms in the anti-symmetric representation of $O(N)$, is a singlet under $(-1)^{F}$, and does not transform under time-reversal when $q \bmod 4=2 \cdot{ }^{12}$ If we proceed by first integrating-out the coupling $J_{i_{1} i_{2} \ldots i_{q}}$, we can easily show that $\left\langle\mathcal{O}_{A}\right\rangle=0$ since there is no spontaneous breaking of the global symmetry in $1 D$. However, when studying the correlator which have primarily discussed in section 3 : $:\left|\left\langle\mathcal{O}_{A}\right\rangle\right|^{2}$ :, we can no longer use symmetry arguments to show that this correlator vanishes. To obtain : $\left|\left\langle\mathcal{O}_{A}\right\rangle\right|^{2}$ : we need to consider two copies of the SYK model, coupled through the averaging of the same random coupling:

$$
\begin{aligned}
:\left|\left\langle\mathcal{O}_{A}\right\rangle\right|^{2}: \sim \int d J_{i_{1} i_{2} \ldots i_{q}} & \int D \psi_{i}^{L} D \psi^{R}\left(\psi_{[i}^{L} \psi_{j]}^{L}\right)\left(\psi_{[i}^{R} \psi_{j]}^{R}\right)^{\dagger} \\
& \times e^{-\int d \tau \sum_{P \in\{L, R\}}\left[\sum_{i=1}^{N} \psi_{i}^{P} \partial_{\tau} \psi_{i}^{P}-(i)^{\frac{q}{2}} J_{i_{1} \ldots i_{q}} \psi_{i_{1}}^{P} \ldots \psi_{i_{q}}^{P}\right]} .
\end{aligned}
$$

We can now perform the same or different $O(N)$ transformations on the $\mathrm{L}$ and $\mathrm{R}$ fields. If we perform the same transformation, the operator that we have inserted is invariant and the transformation of the coupling $J_{i_{1} i_{2} \ldots i_{q}}$ remains the same as the one described above; in such a case the path integral in (3.23) remains unchanged after the transformation and there is no reason why : $\left|\left\langle\mathcal{O}_{A}\right\rangle\right|^{2}$ : should vanish. If we perform different transformations on the $\mathrm{L}$ and $\mathrm{R}$ fields then the path integral over the two SYK copies no longer has an emergent $O(N)$ symmetry since there is no way to act with a unique $O(N)$ transformation on $J_{i_{1} i_{2} \ldots i_{q}}$ which would leave the path integral invariant. Thus, we find that : $\left|\left\langle\mathcal{O}_{A}\right\rangle\right|^{2}$ :, as compared to $\left\langle\mathcal{O}_{A}\right\rangle$, is not protected by any symmetry when considering the ensemble average.

\footnotetext{
${ }^{12}$ The factor of $i$ is important in order for $\mathcal{O}_{A}$ to be Hermitian. This will be important when discussing how time-reversal acts on $\mathcal{O}_{A}$ in SYK models with $q \bmod 4=0$.
} 
We can also rephrase the above result by coupling the $O(N)$ charge in SYK, $Q_{i j}^{O(N)}=$ $\psi_{[i} \frac{d}{d t} \psi_{j]}$ transforming in the anti-symmetric representation of $O(N)$, to a background gauge field $A^{i j}$, i.e. by adding $A^{i j} \psi_{[i} \frac{d}{d t} \psi_{j]}$ to the Lagrangian in 3.22. If we consider multiple copies of the system, with a unique random coupling $J_{i_{1} i_{2} \ldots i_{q}}$ as in (3.23), we have to also introduce separate copies of the background gauge field (for instance, $A_{L}$ and $A_{R}$ when considering two copies). Then, the path integral is only invariant under gauge transformation that are identical between the left and right copies, $A_{L, R} \rightarrow h^{-1} A_{L, R} h+h^{-1} d h$, instead of the most general gauge transformation $A_{L, R} \rightarrow h_{L, R}^{-1} A_{L, R} h_{L, R}+h_{L, R}^{-1} d h_{L, R}$. This rephrasing emphasizes that the $O(N)$ symmetry, or more generally, any symmetry emergent after ensemble averaging, cannot be dynamically gauged.

We can contrast the above discussion with the case in which we study correlators of an operator that is charged under $O(N)$ but is also charged under some discrete symmetry which is present in each SYK instance. For instance, we can consider $\mathcal{O}_{V}=\psi_{i}$ which is charged under the vector representation of $O(N)$ but is also charged under $(-1)^{F}$ in any instance of the q-state SYK model 13 Alternatively, we can consider the operator the same operator as above, $\mathcal{O}_{A}=i \psi_{[i} \psi_{j]}$ in SYK models with $q \bmod 4=0$ where $\mathcal{O}_{A}$ transforms non-trivially under time-reversal. ${ }^{14}$ Since neither time-reversal nor $(-1)^{F}$ are broken, we conclude that $\left\langle\mathcal{O}_{A, V}\right\rangle=0$ in each instance of the SYK model; consequently, following from the path integral construction analogus to 3.23 , we conclude that : $\left|\left\langle\mathcal{O}_{A, V}\right\rangle\right|^{2}:=0$.

Thus, if imagine the bulk dual of the SYK model, $(-1)^{F}$ and $\mathcal{T}$ correspond to bulk gauge symmetries and the operators $\mathcal{O}_{V}$ and $\mathcal{O}_{A}$ (for $q \bmod 4=0$ ) are charged under the corresponding bulk gauge fields. The latter corresponds to summing over both orientable and unorientable manifolds, while the former corresponds to summing over spin structures [55]. On the other hand, at the level of the low energy effective action, the bulk has an $O(N)$ bulk global symmetry whose charge conservation is explicitly violated by the contribution of replica wormholes. 15

Another useful model which illustrates the phenomenon discussed above is the matrix dual of JT with $\mathbb{Z}_{k}$ BF theory discussed in section 3.2 .2 . Due to the bulk gauge $\mathbb{Z}_{k}$ symmetry, the dual matrix model will have an exact global $\mathbb{Z}_{k}$ symmetry. This means the Hilbert space can be decomposed into sectors with different representations, which can be labelled by

\footnotetext{
${ }^{13}$ In those models, we have that $(-1)^{F} \mathcal{O}_{V}(t)(-1)^{F}=-\mathcal{O}_{V}(t)$.

${ }^{14}$ In Lorentzian signature, for models with $q \bmod 4=0$, we have that $\mathcal{T} \psi_{i}(0)=\psi_{i}(0)$ from which it follows that $\mathcal{T} \mathcal{O}_{A}(t) \mathcal{T}^{-1}=-\mathcal{O}_{A}(-t)$. A detailed discussion of the action of time-reversal is given in [55].

${ }^{15}$ In the SYK model, there is also a $\frac{1}{N^{q}}$ violation of $O(N)$ symmetry, in addition to the non-perturbative corrections we are talking about.
} 
$r \in \mathbb{Z}_{k}$ [48, 49]:

$$
\mathcal{H}=\bigoplus_{r} \mathcal{H}_{r} .
$$

The Hamiltonian will be block diagonal in the basis of different representation $r$ :

$$
H=\left(\begin{array}{cccc}
H_{1} & & & \\
& H_{2} & & \\
& & \ldots & \\
& & & H_{k}
\end{array}\right)
$$

where $H_{1}, \ldots, H_{k}$ are independent random matrices of the same dimension. In this context, the boundary global $\mathbb{Z}_{k}$ symmetry, which corresponds to the bulk gauge symmetry, is given by $Q=\operatorname{diag}(1, \ldots, 1,2, \ldots, 2, \ldots, k, \ldots, k)$ for which $[H, Q]=0$ and the $\mathbb{Z}_{k}$ generator is

given by $e^{\frac{2 \pi i Q}{k}}$. The $r$-sector carries charge $r$ under the one-form symmetry that transforms the Wilson line in the corresponding representation.

We now discuss the meaning of the zero-form bulk global $\mathbb{Z}_{k}$ symmetry on the boundary. On the boundary this corresponds to the $\mathbb{Z}_{k}$ permutation of the different sectors, i.e. $H_{i} \rightarrow$ $H_{(i+1) \bmod k}$, which is an emergent symmetry after taking ensemble average.

It is also useful to take the opposite perspective. Suppose that we have a bulk gravitational theory which has a low energy effective theory with a global symmetry ${ }^{16}$ Then, if we include the contributions of wormholes connecting different boundaries, we will encounter the factorization puzzle discussed in [17, 39]: for instance, the partition function with two boundaries does not factorize. One resolution of this puzzle is that the boundary dual is an ensemble average of theories. In this case, the bulk global symmetry will necessarily arise as an emergent symmetry on the boundary after taking the ensemble average. If we want to restore factorization in the bulk by finding a UV completion of the theory (in which case we assume that the bulk is dual to a single instance of the ensemble), this inevitably requires an explicit breaking of all bulk global symmetries.

\section{Charged state reconstructions}

\subsection{General argument}

While the past computations made it clear that correlators in the ensemble do not obey naive charge conservation properties, and therefore states with different charges are not necessarily

\footnotetext{
${ }^{16}$ For instance, we can consider this low-energy effective theory to be given by $\mathcal{N}=8$ supergravity, which has an $S U(8)$ global symmetry.
} 
orthogonal. However, we have not yet made contact with the argument of Banks and Seiberg regarding the entropy of remnants [6]. In order to better relate our analysis to that of [6], we would like to understand whether there are indeed a large or infinite number (depending on the dimensions of unitary irreducible representations of $G$ ) of remnant states that are indistinguishable. Here, we will rather show that when including the contribution of all connected geometries to the gravitational path integral, the black hole and remnant states are spanned by a finite, but large, basis of charged states. ${ }^{17}$

For simplicity, we will consider excitations that carry charge of $U(1)$ symmetry inside the horizon of a thermofield double state, and the extension to the one-side black hole case or to other global symmetry groups is straightforward. Let's consider a candidate of $K$ charged states $\left\{\left|q_{1}\right\rangle, \ldots,\left|q_{K}\right\rangle\right\}$, created by acting with the operators $\mathcal{O}_{q_{j}}$ that carry $U(1)$ charge $q_{j}$ on the thermofield double state at the middle of the Euclidean evolution. We would like show that any other interior state $|\psi\rangle=\mathcal{O}_{\psi}|H H\rangle$ (see equation (3.1)), for some arbitrary operator $\mathcal{O}_{\psi}$, can be reconstructed as a linear superposition of the states $\left|q_{i}\right\rangle$ for $K>e^{2 S_{\mathrm{BH}}}$. This amounts to showing that $|\psi\rangle$ can be written as, ${ }^{18}$

$$
|\psi\rangle=\sum_{i=1}^{K} f^{i}\left|q_{i}\right\rangle
$$

for some complex coefficients $f^{i}$. To simplify our equations a bit in later discussions, we shall use short-hand notation $\left|q_{f}\right\rangle \equiv \sum_{i=1}^{K} f^{i}\left|q_{i}\right\rangle$ to represent the state with arbitrary $f^{i}$.

Our goal is then to maximize the overlap between $|q\rangle$ and $\left|q_{f}\right\rangle$ :

$$
: \max _{f^{i}} \frac{\left\langle q_{f} \mid \psi\right\rangle}{\sqrt{\langle\psi \mid \psi\rangle\left\langle q_{f} \mid q_{f}\right\rangle}}:=: \sum_{i=1}^{K}\left(f^{i}\right)^{*} \frac{\left\langle q_{i} \mid \psi\right\rangle}{\sqrt{\langle\psi \mid \psi\rangle\left\langle q_{f} \mid q_{f}\right\rangle}}:
$$

where we use the notation : $\cdots$ : to signal at which point we are taking the ensemble average if preparing the gravitational state in a system which has a boundary dual. The fidelity between these two states is equal to the absolute value squared of the inner product and is strictly less or equal to one. Therefore, if we can show that for any state $|q\rangle$ the overlap can be arbitrarily close to 1 with some choice of $f^{i}$, this will mean that the $K$ charged states span a complete basis inside the black hole horizon.

\footnotetext{
${ }^{17}$ We acknowledge Arvin Shahbazi Moghaddam and Douglas Stanford for useful discussions and suggestions on this topic.

${ }^{18}$ The computation could in principle be generalized to start with different orthogonal states in the QFT, not necessarily with different charges. However, when starting with the charged states $\left|q_{i}\right\rangle$, we do not need to use any dynamical data about higher-point functions in the theory beyond the geodesic approximation. We hope to revisit this calculation in more general QFTs in future work.
} 
To find the maximum value of the overlap, we can first introduce a Lagrange multiplier $\lambda$ to impose the normalization constraint for $\left|q_{f}\right\rangle$ and then maximize:

$$
: \max _{f^{i}, \lambda}\left[\sum_{i}\left(f^{i}\right)^{*}\left\langle q_{i} \mid \psi\right\rangle-\lambda\left(1-\sum_{i, j}\left(f^{i}\right)^{*} f^{j}\left\langle q_{i} \mid q_{j}\right\rangle\right)\right]:
$$

Extremizing equation 4.3 with respect to $f^{i}$ and $\lambda$ we find:

$$
(f)^{*} M f=1, \quad V=\lambda M f, \quad \text { from which, } \quad \lambda=\sqrt{V M^{-1} V}, \quad f=\frac{M^{-1} V}{\sqrt{V M^{-1} V}},
$$

where we have used the simplified vector notation $M_{i j}=\left\langle q_{i} \mid q_{j}\right\rangle$ and $V_{i}=\left\langle\psi \mid q_{i}\right\rangle$. This result implies that the maximum overlap between $|q\rangle$ and $\left|q_{f}\right\rangle$ is given by:

$$
: \frac{\max _{f^{i}} \sum_{i} f^{i}\left\langle\psi \mid q_{i}\right\rangle}{\sqrt{\langle\psi \mid \psi\rangle}}:=\frac{: \sqrt{V M^{-1} V}:}{\sqrt{\langle\psi \mid \psi\rangle}}=\frac{: \sqrt{\left\langle\psi \mid q_{i}\right\rangle\left(\left\langle q_{i} \mid q_{j}\right\rangle\right)^{-1}\left\langle q_{j} \mid \psi\right\rangle}:}{\sqrt{\langle\psi \mid \psi\rangle}},
$$

where we have already normalized $\left|q_{f}\right\rangle$ to have unit norm.

To evaluate this on the gravitational side, we will use the replica trick by first considering the quantity : $V M^{n} V$ : and then analytically continue to $n=-1$. Since we will show that $\frac{: V M^{-1} V}{\langle\psi \mid \psi\rangle}$ is arbitrarily close to 1 , it will then follow that $\frac{: \sqrt{V M^{-1} V}}{\sqrt{\langle\psi \mid \psi\rangle}}$ is also arbitrarily close to 1 . We would thus like to first evaluate the ensemble average for the function under the square root

$$
:\left\langle\psi \mid q_{i}\right\rangle\left(\left\langle q_{i} \mid q_{j}\right\rangle\right)^{n}\left\langle q_{j} \mid \psi\right\rangle:=: \sum_{p_{1}, \ldots, p_{n+1} \in\left\{q_{1}, \ldots, q_{K}\right\}}\left\langle\psi \mid p_{1}\right\rangle\left\langle p_{1} \mid p_{2}\right\rangle \ldots\left\langle p_{n} \mid p_{n+1}\right\rangle\left\langle p_{n+1} \mid \psi\right\rangle:,
$$

with $n \in \mathbb{Z}$, using the Euclidean path integral in gravity. This requires us to sum over all possible geometries with $n+2$ asymptotic boundaries, with two charged operator insertions on each boundary, i.e. $\mathcal{O}_{\psi}^{\dagger}$ and $\mathcal{O}_{p_{1}}$ on the first, $\mathcal{O}_{p_{1}}^{\dagger}$ and $\mathcal{O}_{p_{2}}$ on the second, and so on, up to $\mathcal{O}_{p_{n+1}}^{\dagger}$ and $\mathcal{O}_{\psi}$ on the last.

This is essentially the same type of calculation in the page curve of an evaporating black hole or in the Petz map reconstruction [16, 15]. In particular, in the limit of $K>e^{2 S_{\mathrm{BH}}}$, the dominating geometry will be the fully connected pinwheel geometry in figure 3 . In such a configuration, every operator $\mathcal{O}_{i}$ is connected with $\mathcal{O}_{i}^{\dagger}$ through a bulk propagator. Summing over all the $i$ indices leads to the maximum power of $K$ :

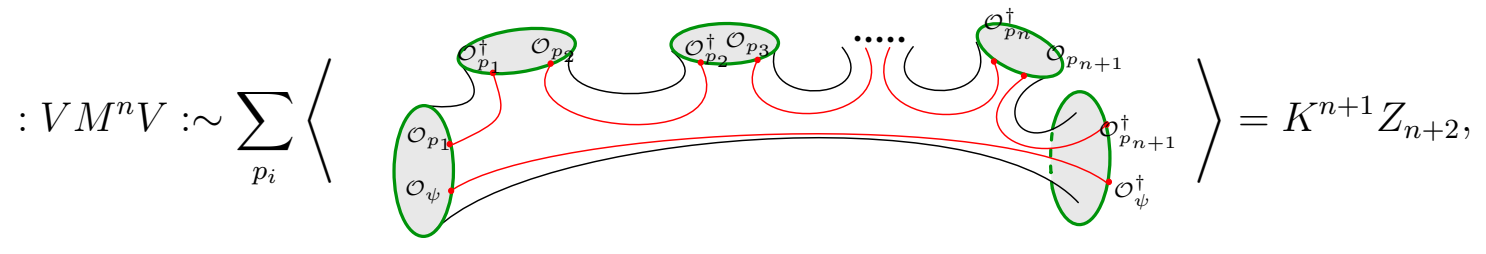



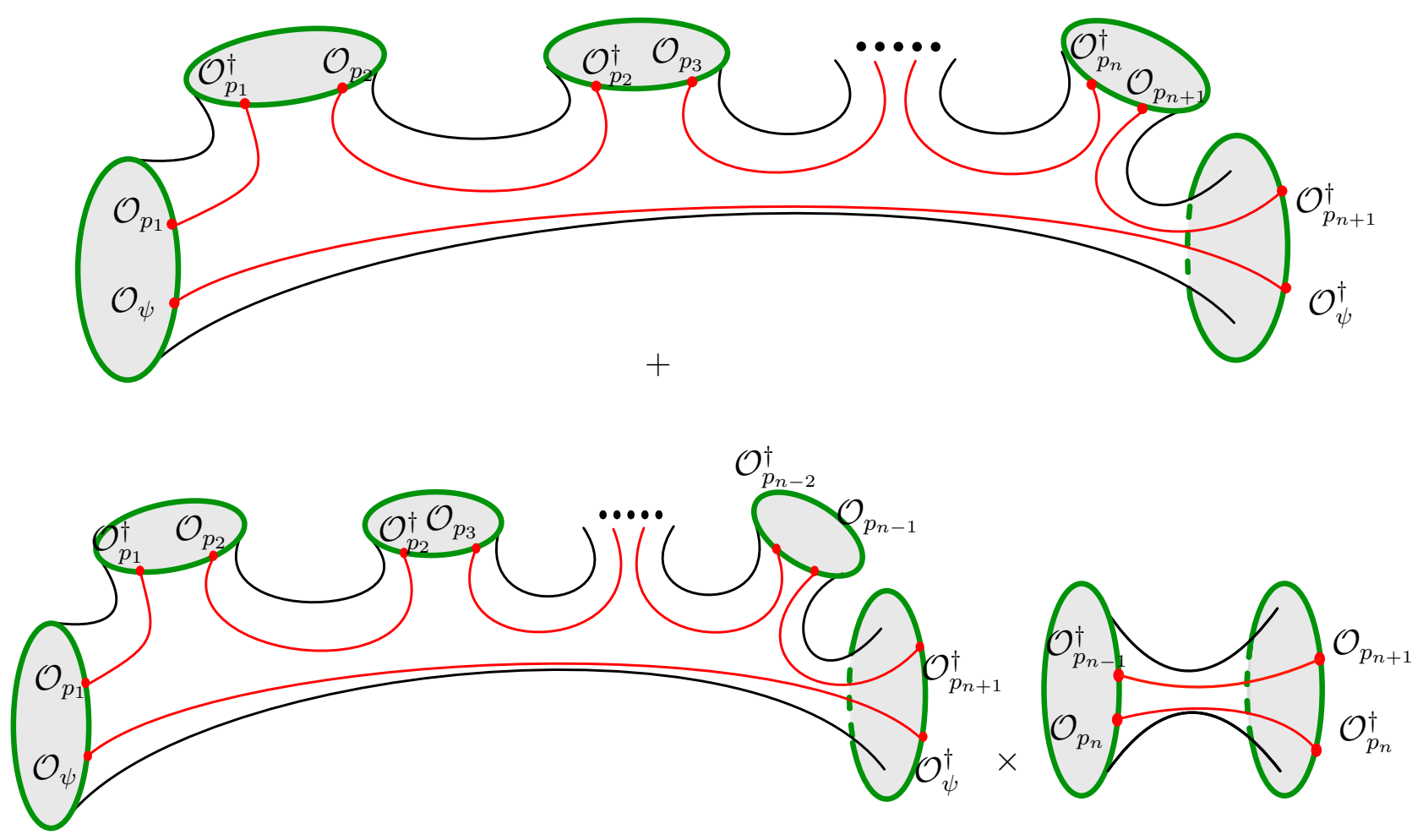

Figure 3: The first line shows the leading order contributions when evaluation the matrix $M^{n}$ needed in order to find null states. The second line shows an example of a subleading in $K$ contribution which is only present when $p_{n-1}=p_{n+1}$. When the basis set of states, given by the charges $p_{j} \in\left\{q_{1}, \ldots, q_{K}\right\}$, has a dimension $K>e^{2 S_{\mathrm{BH}}}$, the leading contribution is solely given by the first geometry with all other contributions suppressed in $k$.

where $Z_{n+2}$ is the gravitational path integral over the pinwheel geometry with $n+2$ boundaries and insertion of $n+2$ bulk propagators as shown in the figure.

Analytically continuing this result to the limit of $n=-1$, we directly recover the inner product of $\langle\psi \mid \psi\rangle$ :

$$
\left\langle q_{f} \mid \psi\right\rangle=: V M^{-1} V: \sim\langle\overbrace{}^{\mathcal{O}_{\psi}^{\dagger}}\rangle=\langle\psi \mid \psi\rangle,
$$

which means we have succeeded in reconstruction state $|\psi\rangle$ with states $\left\{\left|q_{i}\right\rangle\right\}$.

It is straightforward to see that the above argument continues to hold for more general 
interior states $|\psi\rangle$ and $\left|q_{i}\right\rangle$ beyond theories with the existence of global symmetry since the main property we are using is that taking $K$ large prefers the completely connected geometry. Consequently, this implies that any excitations inside the black hole horizon (including the arbitrary state $|\psi\rangle$ ) can be rewritten as a linear combination of $K$ other states $\left\{\left|q_{1}\right\rangle, \ldots,\left|q_{K}\right\rangle\right\}$ for $K>e^{2 S_{\mathrm{BH}}}$ (or $e^{S_{\mathrm{BH}}}$ for a single-sided black hole).$^{19}$ Notice that although we are drawing two dimensional figures, this argument should hold for higher dimensional gravitational theories. As long as we take the dimension of the basis, $K$, to be sufficiently large, than the connected geometry dominates over all other in the Euclidean path integral. This is sufficient if we wish to find the analytic continuation of : $V M^{-1} V:$ from 4.8. With our new understanding of the over-completeness of charged states in the gravitational theory, in the next section we will re-analyze the fate of remnants in the argument by Banks and Seiberg, reviewed in section 2.2. For now however, we analyze possible corrections to 4.8 .

\subsection{Planar Resummations in JT gravity}

In this section, we discuss the state reconstruction in JT gravity. First we notice that as long as $K \gg 1$ and $e^{S_{0}} \gg 1$, the dominating contribution to : $V M^{n} V$ : are the planar geometries just like in the case of the end of the world (EoW) brane model, studied in [15] (See also [58, 59, 60]). Therefore, we can use the same resolvent technique to solve this exactly. The strategy will be first consider insertion of the resolvent operator $\mathbf{R}$ of $M$ :

$$
: V \mathbf{R}(\lambda) V: \equiv: V \frac{1}{\lambda-M} V:, \quad: V M^{n} V:=\frac{1}{2 \pi i} \oint d \lambda \lambda^{n}: V \mathbf{R}(\lambda) V:,
$$

and then do an analytic continuation to $n=-1$. Due to the typical branch cut structure of the resolvent, the integration contour needs to be deformed and we will see how that precisely works. The boundary condition of : $V \mathbf{R}(\lambda) V:$ corresponds to a infinite summation of an indefinite number of boundary circles:

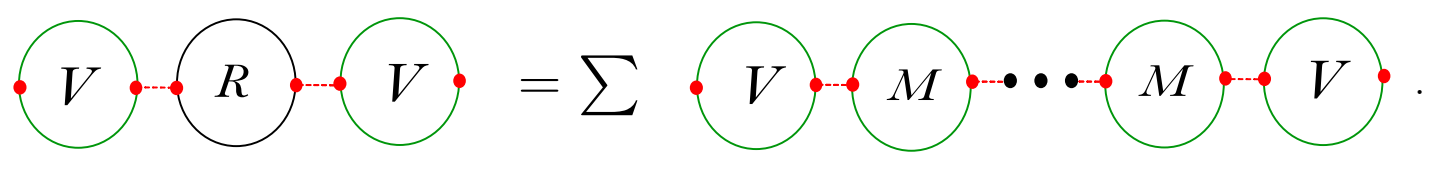

${ }^{19}$ Because of the arguments above we can construct the state

$$
|\omega\rangle=|\psi\rangle-\left|q_{f}\right\rangle
$$

which is null. This is similar in spirit to the existence of null-states recently discussed in the context of $\alpha$-states in [57]. However, our interpretation for the Hilbert space of the theory is different than that in [57. More explicitly, the Hilbert space containing the states in (4.1) is not the baby-universe Hilbert space considered in [57]; rather, it is the state obtained by acting with charged operators on the gravitational Hartle-Hawking state. 
The corresponding bulk geometries can be classified by the geometry $\mathcal{M}$ that connects the two $V$ type boundaries, which must exist for the answer to be nonzero. To specify $\mathcal{M}$, we also need to know how $\mathcal{M}$ ends on the various $M$ type boundaries and we will use the notation $\mathcal{M}_{2, l}$ to represent the geometry that ends on $l$ such $M$ type boundaries. Together with the two $V$ type boundaries, $\mathcal{M}_{2, l}$ has total $l+2$ boundaries. Between these boundaries we still have an infinite sum of planar geometries which can be rewritten as an insertion of a resolvent:

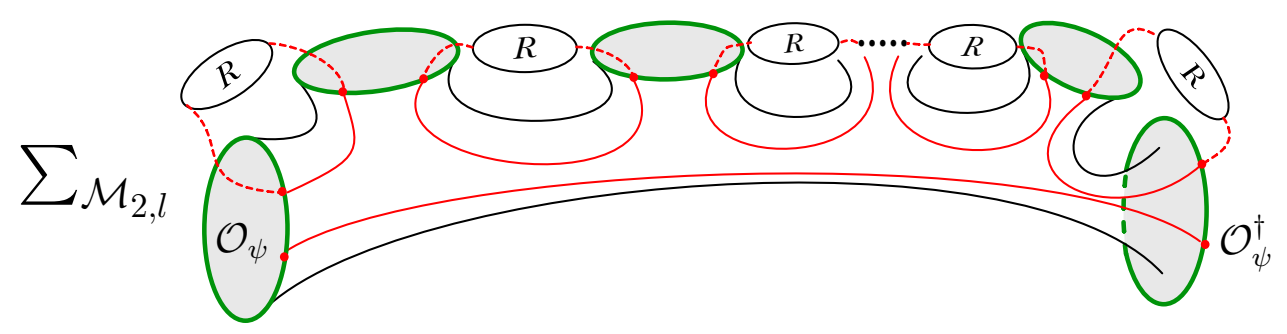

Summing over all the $\mathcal{M}_{2, l} \mathrm{~s}$, we get an exact expression of : $V \mathbf{R}(\lambda) V:$ in terms of the resolvent $R(\lambda) \equiv \operatorname{Tr} \mathbf{R}(\lambda)$ :

$$
: V \mathbf{R}(\lambda) V:=\sum_{l=0}^{\infty} R^{l+1}(\lambda) Z_{l+2}
$$

where we used the notation $Z_{l+2}$ to represent the gravitational path integral over the geometry $M_{2, l}$ (this is the same notation as in equation 4.7). The gravitational path integral $Z_{l+2}$ in JT can be derives using the same cutting and gluing procedure we used in section 3.2.1. By cutting along the propagators, we can separate the pinwheel geometry into two pieces, each contains $l+2$ numbers of the geodesics $\ell_{i}$ and the semi-boundaries $\beta_{i}=\frac{\beta}{2}$. Since there is no additional operators insertion in such geometry, all the boundaries have the same energy and the full gravitational path integral can be written as:

$$
\int d E \rho(E) \prod_{i} e^{-\beta_{i} E}\left\langle E \mid \ell_{i}\right\rangle .
$$

Gluing two copies of this geometry together with the weighting of the propagators $e^{-m \ell_{i}}$, we get:

$$
\begin{aligned}
Z_{n} & =e^{S_{0}(2-n)} \int d E_{1} d E_{2} \rho\left(E_{1}\right) \rho\left(E_{2}\right) e^{-\frac{n}{2} \beta\left(E_{1}+E_{2}\right)} \prod_{i} \int d \ell_{i}\left\langle E_{1} \mid \ell_{i}\right\rangle e^{-m \ell_{i}}\left\langle\ell_{i} \mid E_{2}\right\rangle \\
& =\int d E_{1} d E_{2} e^{2 S_{0}} \rho\left(E_{1}\right) \rho\left(E_{2}\right) y_{E_{1}, E_{2}}^{n} ; \quad y_{E_{1}, E_{2}}=e^{-S_{0}} e^{-\frac{1}{2} \beta\left(E_{1}+E_{2}\right)}\left\langle E_{1}|O O| E_{2}\right\rangle .
\end{aligned}
$$

We can understand this formula as a Boltzmann summation over the product of $l+2$ correlators in the energy basis $E_{1,2}$, and we expect that this is a general result, that holds beyond 
JT gravity. On the other hand, if we go to microcanonical ensemble rather than canonical ensemble, the result of the path integral will just be the integrand. Plugging this in equation (4.13), and summing over the geometric series, one gets:

$$
\begin{gathered}
: V \mathbf{R}(\lambda) V:=e^{2 S_{0}} \int d E_{1} d E_{2} \rho\left(E_{1}\right) \rho\left(E_{2}\right) \frac{y^{2} R}{1-y R} \\
\left\langle q_{f} \mid \psi\right\rangle=: V M^{-1} V:=e^{2 S_{0}} \int d E_{1} d E_{2} \rho\left(E_{1}\right) \rho\left(E_{2}\right) y \oint \frac{d \lambda}{2 \pi i \lambda} \frac{y R}{1-y R} .
\end{gathered}
$$

Therefore, if we know the value of the resolvent $R$, we know the full answer of $: V \mathbf{R} V:$ and also : $V M^{-1} V$ :. Using free probability theory, or by classifying the planar diagrams as what we did before, one finds that the resolvent satisfies a Schwinger-Dyson (SD) equation ([15]):

$$
\lambda R=K+\sum_{n=1} Z_{n} R^{n}=K+\int d E_{1} d E_{2} e^{2 S_{0}} \rho\left(E_{1}\right) \rho\left(E_{2}\right) \frac{y R}{1-y R} .
$$

The physics of this Schwinger-Dyson equation is quite rich, and it includes the phase transition between different Renyi entropies of the system; generally there is no known exact method of solving this equation, apart from numerics [15]. Below we consider a simpler equation by directly going to the microcanonical ensemble and then draw some general lessons from that. We also present the computation in the canonical ensemble in appendix B.

Going to the microcanoincal ensemble, we are fixing the energy of $E_{1,2}$ into a small energy window $(E, E+\delta E)$. This leads to a simplified version of equation (4.16) and equation 4.17):

$$
\begin{gathered}
: V \mathbf{R}(\lambda) V:=e^{2 \mathbf{S}} \frac{y^{2} R}{1-y R}, \\
\lambda R=K+e^{2 \mathbf{S}} \frac{y R}{1-y R},
\end{gathered}
$$

where we used the notation $e^{\mathbf{S}} \equiv \delta E e^{S_{0}} \rho(E)$ and $Z_{n}=e^{2 \mathbf{S}} y^{n}$. Solving the quadratic equation 4.19), we get:

$$
\begin{aligned}
& R(\lambda)=\frac{1}{2 y}+\frac{K-e^{2 \mathbf{S}}}{2 \lambda}-\frac{1}{2 \lambda y} \sqrt{\left(\lambda-\lambda_{+}\right)\left(\lambda-\lambda_{-}\right)} ; \quad \lambda_{ \pm}=y\left(e^{\mathbf{S}} \pm \sqrt{K}\right)^{2}, \\
& D(\lambda)=\frac{1}{2 \lambda y} \sqrt{\left(\lambda_{+}-\lambda\right)\left(\lambda-\lambda_{-}\right)}+\delta(\lambda)\left(K-e^{2 \mathbf{S}}\right) \theta\left(K-e^{2 \mathbf{S}}\right),
\end{aligned}
$$

where $D(\lambda) \equiv \frac{1}{2 \pi i}(R(\lambda-i \epsilon)-R(\lambda+i \epsilon))$ is the density of states. We see that when $K<e^{\mathbf{S}}$, the density of state is fully support at $\left(\lambda_{-}, \lambda_{+}\right)$. After $K>e^{\mathbf{S}}$, the support of $D(\lambda)$ split into two parts: there are $K-e^{2 \mathbf{S}}$ states located at $\lambda=0$ and $e^{2 \mathbf{S}}$ distributed between $\lambda_{-}$ and $\lambda_{+}$. 
Finally, let's look at the inner product $\left\langle q_{f} \mid \psi\right\rangle$. Combining equation 4.18, with equation 4.19, we have:

$$
: V \mathbf{R}(\lambda) V:=(\lambda R-K) y
$$

which has a branch cut coming from the resolvent. This gives us:

$$
: V M^{-1} V:=\lim _{n \rightarrow-1} \oint d \lambda \lambda^{n} V \mathbf{R}(\lambda) V=\lim _{n \rightarrow-1} \frac{1}{2 \pi i} \oint d \lambda \lambda^{n}(\lambda R(\lambda)-K) y
$$

When we do the analytic continuation in $n, \lambda^{n+1}$ will generically have a branch cut from 0 to infinity. To avoid this issue, we can first deform the integration contour of $\lambda$ to go around the branch cut of $R(\lambda)$ and then analytically continue in $n$. This leads to the final expression:

$$
\left\langle q_{f} \mid \psi\right\rangle=: V M^{-1} V:=y \int_{\lambda_{-}}^{\lambda_{+}} d \lambda D(\lambda)=\left\{\begin{array}{ll}
K y & K<e^{2 \mathbf{S}} \\
e^{2 \mathbf{S}} y & K \geq e^{2 \mathbf{S}}
\end{array} .\right.
$$

Recall that $\langle\psi \mid \psi\rangle=Z_{1}=e^{2 \mathbf{S}} y$, we see that the inner product between $|\psi\rangle$ and $\left|q_{f}\right\rangle$ is equal to one when $K$ is bigger than $e^{2 \mathbf{S}}$. This means in the microcanoincal ensemble case, any state $|\psi\rangle$ can be reconstructed from the $K=e^{2 \mathbf{S}}$ charge states $\left\{\left|q_{1}\right\rangle, \ldots\left|q_{K}\right\rangle\right\}$. In other words, they form a complete basis of the states in the microcanonical black hole.

\section{The fate of remnants}

In section 4, we argue that states that are linearly independent in a quantum field theory can become over-complete when this theory is coupled to gravity. In particular, we showed that when coupling a theory with global symmetry to gravity, then a generic excitation inside of a black hole $|\psi\rangle$ can be written as a linear combination of a basis of $K$ charged states, $\left\{\left|q_{1}\right\rangle, \ldots,\left|q_{K}\right\rangle\right\}$, when $K$ is larger than $e^{2 S_{\mathrm{BH}}}$ (or $e^{S_{\mathrm{BH}}}$ for a single sided black hole). While the explicit computation in section 4, was primarily done for charged excitations in the thermal-field double state, a similar computation should apply to the states inside of an evaporating black hole, which can be modeled by coupling the system shown in figure 3 to a bath, represented by a large region in flat-space, where gravitational effects can be ignored (for instance, see [36]).

As in [6], we can imagine that the gravitational effective theory which we use in the path integral computation can be trusted up to the point where a remnant is formed. That is the radius of the black hole horizon is given by $r_{h}=X L_{\mathrm{Pl}}$ and its Bekenstein-Hawking entropy

is given by $e^{S_{\mathrm{BH}}}=e^{\pi X^{2}}$. In the argument made in [6], each remnant state that carried the representation $R$ of the global symmetry was considered to be indistinguishable and 


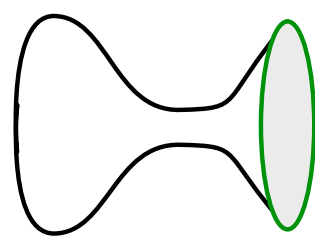

Black hole at late times
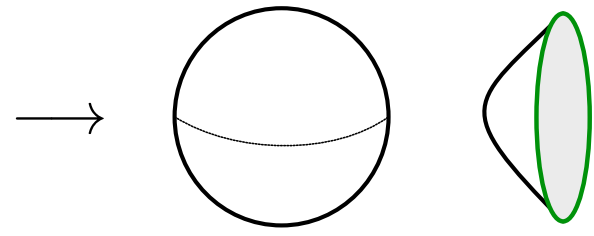

Closed universe

Figure 4: Spatial section of black hole at late times, decaying into a closed universe.

independent. This leads to a large degeneracy of remnant states which, with some technical assumptions mentioned in section 2.2, contradicted the covariant entropy bound, or its more refined version: the "central dogma" that black hole describes a quantum mechanics system with $S_{\mathrm{BH}}$ degrees of freedom. However, because we have found that by considering the contribution of replica wormholes, the Hilbert space in the gravitational theory is overcomplete, the situation is now much less dramatic. The complete basis of the states inside of the Remnant can be chosen to be the $K$ charge states. Since $K$ is order $e^{S_{\mathrm{BH}}}$, this means that the degree of freedom of the remnant is given by $\log K \sim \pi X^{2}$ in consistent with the "central dogma".20

We can now speculate about the ultimate fate of these remnants. Historically, remnants were argued to be non-existent due to the thermodynamic instability caused by the large internal entropy [32, 33]. From our discussion, the possible remnants forming from black hole evaporation do not encounter such issues since the entropy still obeys the BenkensteinHawking bound and can, in principle, exist in nature. However, it might no longer be appropriate to call such an object a remnant since most information of the black hole has "escaped" through the replica wormhole and is encoded in the Hawking radiation. For instance, information about the global symmetry charge of the initial black hole is no longer captured by the remnant states. Because of that, if such objects exist at the end of evaporation, perhaps a better name for them is "faint remnants".

We also see that whether or not the remnant is formed from global symmetry charged states does not make a difference due to the large violation of global symmetry inside the remnant. From an outside observer, the remnant seems to be nothing more special than an ordinary small black hole and it seems reasonable that it will eventually decay into a closed universe including the whole interior of the black hole, as portrayed in figure 4.

Extrapolating our result in section 4 to the closed universe case, we conclude that all the

\footnotetext{
${ }^{20}$ Notice that there could be order one correction to the Bekenstein-Hawking entropy. For instance, it is well known that the black hole entropy receives $\sim \log r_{h}^{2}$ corrections with an unfixed sign.
} 

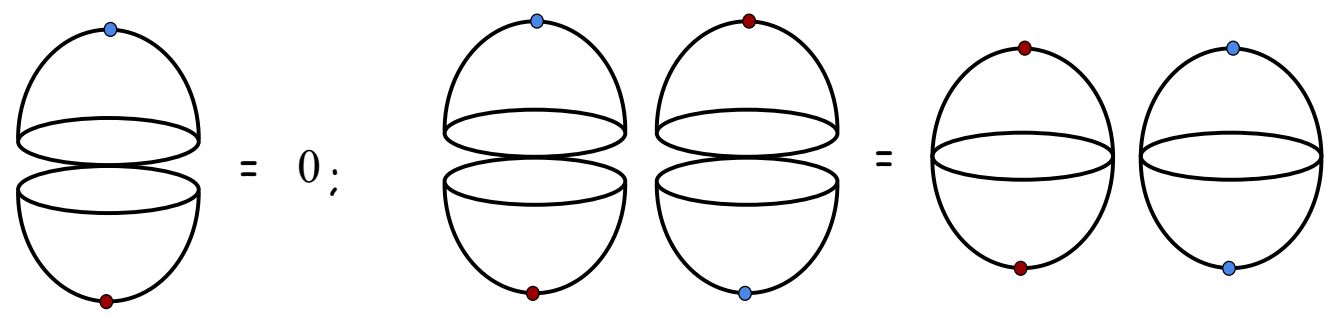

Figure 5: The inner product between different states in a closed universe. The brown and blue dots represent insertion of operators with different charges. On the left figure, the inner product between these two states is zero. On the right figure, the fidelity of these two states is equal to one, which means the two states are equivalent up to a phase.

different states inside the closed universe are equivalent up to a random phase:

$$
\left|q_{i}\right\rangle_{\mathrm{CU}}=e^{i \theta_{i}}|\mathrm{CU}\rangle
$$

This is consistent with the following gravitational picture shown in figure 5 , the states $\left|q_{i}\right\rangle$ and $\left|q_{j}\right\rangle$ in a closed universe can be prepared by inserting different operators in the past. If we calculate their inner product $\left\langle q_{i} \mid q_{j}\right\rangle$ directly in the bulk, the answer will be zero. However, the fidelity between these two states $\left|\left\langle q_{i} \mid q_{j}\right\rangle\right|^{2}$ is equal to one since the closed universe can just connect between the two copies. This is a strong hint that $\left|q_{i}\right\rangle$ and $\left|q_{j}\right\rangle$ are actually the same state up to a phase which is random, so that after ensemble average the inner product vanishes. In other words, our computation suggests that the Hilbert space of a closed universe is one-dimensional.

Using this property, let's reexamine Hawking's original argument (section 2.1) about the charge violation that can occur during black hole evaporation. If the initial state of the universe was in a singlet state, after creating a black hole and letting it evaporate, the rest of the universe will be in a charge singlet state together with the baby universe. Using the relation between the different charged states inside of the closed universe (as in (5.1), we find that the final state will become a random superposition of charged states. Schematically we have:

$$
\mid \text { final state }\rangle=\sum_{i}\left|q_{i}\right\rangle_{\mathrm{CU}}\left|\bar{q}_{i}\right\rangle_{\mathrm{rest}}=\sum_{i} e^{i \theta_{i}}\left|\bar{q}_{i}\right\rangle_{\mathrm{rest}}|\mathrm{CU}\rangle
$$

Once again, to make a connection with Hawking's result, we can consider the density matrix of the final state and perform an ensemble average. This gives us a thermal distribution of the charge sectors due to the randomness of the phases $\theta_{i}$. 


\section{Conclusion and comparison to past arguments}

This work provides a new argument about the violation of exact global symmetries in quantum gravity, using replica wormholes. We argue that the existence of replica wormholes predicts a non-vanishing transition probability (or inner product) between different charged states. This is a non-perturbative violation of global symmetry charge conservation, and it holds even in a theory with no explicit breaking of the global symmetry up to arbitrarily high energy scales.

The main mechanism for this violation stems from the fact that the transition probability involves two copies of the system. The operator insertions can form a singlet on the replica wormhole which connects the two copies, while the leading disconnected contribution vanishes due to the presence of the global symmetry. As a consequence, quantum gravity does not allow super-selection sectors coming from any exact global symmetries. In principle, our computation in the toy model of JT gravity coupled to matter with a $U(1)$ charge can be embedded to describe the scattering probability from baryons to leptons for higher-dimensional near-extremal Reissner-Nordström black holes, coupled to the Standard Model or to some of its extensions that preserve the baryon-lepton number global symmetry ${ }^{21}$ In contrast, if we gauge the global symmetry, then the transition probability between charged states is still zero, even when including the contribution of replica wormholes. The reason for this is that, after we gauge the symmetry, there will be individual global symmetries associated with each of the boundaries which provide a stronger constraint on correlation functions. An alternative point of view is that there will be Gauss's Law constraint on the throat of the wormhole after we couple to a gauge field.

We also argue that the states that are orthogonal in an ordinary quantum field theory can form an over-complete basis when coupling that quantum field theory to gravity. In the context of black holes and their late-time remnants, this leads to a verification of the "central dogma" that such systems have $S_{\mathrm{BH}}=\frac{A}{4 G_{N}}$ degrees of freedom, even when the number of distinct unitary irreducible representations of the global symmetry is large, or infinite. In addition, our calculation indicates that the Hilbert space of a closed universe is one dimensional.

Let us compare the results of this paper to the previous arguments of [12, 6, 8, 9] against global symmetries in quantum gravity (reviewed in section 2). Due to the contribution of

\footnotetext{
${ }^{21}$ Generically, the contribution of higher topology or multi-boundary geometries to the near-horizon path integral is untrustworthy [61]. That is because there are numerous corrections kick-in (such as corrections to the dilaton potential or from Kaluza-Klein modes [62, 63, 61]), before the $O\left(e^{-S_{\mathrm{BH}}}\right)$ corrections coming from higher topologies. However, because the replica wormhole is the leading non-vanishing contribution due to the $U(1)$ global symmetry, the calculation of the scattering probability is now trustworthy.
} 
replica wormholes, our result provides a concrete base for Hawking's intuition that global symmetry charge is not conserved during the process of black hole evaporation, and our computation extends his argument to eternal black holes. Regarding the dollar matrix discussion in section 2, our gravitational computation predicts a non-factorization property of dollar matrix coming from ensemble average:

$$
: \$_{m m^{\prime} ; n n^{\prime}}:=: S_{m n} S_{m^{\prime} n^{\prime}}^{*}: \neq: S_{m n}:: S_{m^{\prime} n^{\prime}}^{*}:
$$

where $: \cdots$ : denotes the point at which we consider an ensemble average.

We also see that at late times black holes in theories with a global symmetry have a much smaller degeneracy, $\sim e^{\frac{A}{4 G_{N}}}$, than naively expected in the absence of replica wormholes [6]. Our results imply that there is no apparent contradiction in the existence of black hole remnants, which, due to the lack of global symmetry charge conservation, could, in principle, fully evaporate.

Finally, in arguing for the violation of global symmetries in quantum gravity, we have not used the extremal island formula as in [9] and rather, we directly use the contribution of replica wormholes. In particular, our computation quantifies how such geometries lead to the violation of global charge conservation and makes direct contact with the possible fate of remnants. Finally, while previous arguments suggested that global symmetries are absent in the effective field theory beyond some energy level, we are able to explain what the presence of global symmetries in the effective gravitational description implies for a holographic theory. We will expand on this below.

Throughout the paper, when the gravitational theory is holographic, we interpret the boundary theory as an ensemble average. From that point of view, the global symmetry in the bulk is a result of ensemble average of the boundary theories, where, in each member of the ensemble, the global symmetry is absent. An analog of this situation is the $O(N)$ symmetry of the SYK model. For each realization, the coupling constant breaks the global symmetry explicitly, but the distribution of the coupling constant is $O(N)$ invariant. This leads to the existence of an emergent $O(N)$ global symmetry after ensemble average. Gauge symmetries in the bulk, on the other hand, are boundary global symmetries that are present in all members of the ensemble, and, in this case, the contribution of replica wormholes does not lead to a violation of charge conservation.

Our wormhole calculation indicates an explicit breaking of global symmetries in the effective Lagrangian of a single instance of the ensemble. This breaking suggests that any UV completion of this effective Lagrangian, which resolves the factorization puzzle, should not have any manifest global symmetries; rather, global symmetries in quantum gravity are a manifestation of ensemble averaging and absence of factorization. 


\section{Acknowledgement}

We thank Juan Maldacena, Arvin Shahbazi Moghaddam, Stephen Shenker and Douglas Stanford for comments on a draft and for helpful discussions. We thank Edgar Shaghoulian for comments on a draft. The work of P.-S. H. is supported by the U.S. Department of Energy, Office of Science, Office of High Energy Physics, under Award Number DE-SC0011632, and by the Simons Foundation through the Simons Investigator Award. LVI was supported in part by the Simons Collaboration on Ultra-Quantum Matter, a Simons Foundation Grant with No. 651440. ZY is supported in part by the Simons Foundation through the It from Qubit Collaboration.

\section{A Squared one- and two-point function}

In this appendix, we review the calculation of the squared one-point and two-point functions in JT gravity [34, 40]. The basic ingredient is the matrix element of the evolution of the two side Hamiltonian $e^{-\beta_{L} H_{L}-\beta_{R} H_{R}}$ in the geodesic basis:

$$
\left\langle\ell\left|e^{-\beta_{L} H_{L}-\beta_{R} H_{R}}\right| \ell^{\prime}\right\rangle=\int d E \rho(E) e^{-\left(\beta_{L}+\beta_{R}\right) E}\langle\ell \mid E\rangle\left\langle E \mid \ell^{\prime}\right\rangle,
$$

where $\rho(E)=\frac{1}{2 \pi^{2}} \sinh (2 \pi \sqrt{2 E})$ is the density of states and $\langle\ell \mid E\rangle=4 K_{2 i \sqrt{2 E}}\left(4 e^{-\ell / 2}\right)$. This can be understood as the gravitational path integral over a rectangular geometry with two geodesic boundaries $\ell, \ell^{\prime}$ and two asymptotic boundaries (figure 6). The other ingredient is the geodesic approximation of the two point function which is simply given by

$$
\left\langle\ell\left|\mathcal{O}_{q} \mathcal{O}_{-q}\right| \ell^{\prime}\right\rangle=e^{-m \ell}\left\langle\ell \mid \ell^{\prime}\right\rangle
$$

This directly gives us the absolute value of the squared one point function:

$$
\left|\left\langle\mathcal{O}_{q}\right\rangle\right|^{2}=\int d \ell e^{-m \ell}\left\langle\ell\left|e^{-\beta_{L} H_{L}-\beta_{R} H_{R}}\right| \ell\right\rangle=\int d E \rho(E) e^{-\left(\beta_{L}+\beta_{R}\right) E}\left\langle E\left|\mathcal{O}_{q} \mathcal{O}_{-q}\right| E\right\rangle
$$

where $\left\langle E\left|\mathcal{O}_{q} \mathcal{O}_{-q}\right| E^{\prime}\right\rangle=\int d \ell\langle\ell \mid E\rangle\left\langle E^{\prime} \mid \ell\right\rangle e^{-m \ell}$ is the two point function in the energy basis:

$$
\left\langle E\left|\mathcal{O}_{q} \mathcal{O}_{-q}\right| E^{\prime}\right\rangle=\frac{\Gamma\left(m \pm i\left(\sqrt{2 E} \pm \sqrt{2 E^{\prime}}\right)\right)}{2^{2 m+1} \Gamma(2 m)}
$$

where the \pm sign means we need to take product of all the four gamma functions coming from different choice of the \pm signs. 
Similarly, the two point function squared can be calculated from gluing two rectangular regions along the two geodesics where the operators are inserted:

$$
\begin{aligned}
\left|\left\langle\mathcal{O}_{q_{1}} \mathcal{O}_{q_{2}}\right\rangle\right|^{2} & =\int d \ell d \ell^{\prime} e^{-m_{1} \ell}\left\langle\ell\left|e^{-\beta_{L 1} H_{L}-\beta_{R 1} H_{R}}\right| \ell^{\prime}\right\rangle e^{-m_{2} \ell^{\prime}}\left\langle\ell^{\prime}\left|e^{-\beta_{L 2} H_{L}-\beta_{R 2} H_{R}}\right| \ell\right\rangle \\
& =\int d E_{1} d E_{2} \rho\left(E_{1}\right) \rho\left(E_{2}\right) e^{-\left(\beta_{L 1}+\beta_{R 1}\right) E_{1}-\left(\beta_{L 2}+\beta_{R 2}\right) E_{2}}\left\langle E_{1}\left|\mathcal{O}_{q_{1}} \mathcal{O}_{-q_{1}}\right| E_{2}\right\rangle\left\langle E_{2}\left|\mathcal{O}_{q_{2}} \mathcal{O}_{-q_{2}}\right| E_{1}\right\rangle .
\end{aligned}
$$

Let's now look at the semiclassical geometry of the squared one point function and two point function. Using the asymptotic approximation of the gamma function:

$$
\Gamma(m+i x) \Gamma(m-i x) \sim 2 \pi x^{2 m-1} e^{-\pi x}, \quad x \gg 1 .
$$

It is easy to see that both $\left|\left\langle\mathcal{O}_{q}\right\rangle\right|^{2}$ and $\left|\left\langle\mathcal{O}_{q_{1}} \mathcal{O}_{q_{2}}\right\rangle\right|^{2}$ are dominated by low energy configurations:

$$
E \sim \frac{m}{\beta_{L}+\beta_{R}}
$$

This energy is linear in temperature and the dimension of the inserted operator. The effective temperature can be derived from the thermodynamics relation:

$$
\beta_{E}=\frac{\sqrt{2} \pi}{\sqrt{E}} .
$$

With this information, we can look at the saddle configuration of the geodesic across the two asymptotic boundaries. The integration of the geodesic length only contributes to the calculation of the two point function in energy basis:

$$
\begin{aligned}
& \int d \ell\langle\ell \mid E\rangle\langle E \mid \ell\rangle e^{-m \ell}=16 \int d \ell e^{-m \ell} K_{2 i \sqrt{2 E}}^{2}\left(4 e^{-\ell / 2}\right) \\
& =4 \int d \ell d t_{1} d t_{2} e^{-m \ell-2 i \sqrt{2 E}\left(t_{1}+t_{2}\right)-4 e^{-\ell / 2}\left(\cosh t_{1}+\cosh t_{2}\right)}
\end{aligned}
$$

where we use the integral representation of the Bessel K function. The saddle point equations are:

$$
4 e^{-\ell_{*} / 2} \cosh t=m ; \quad 4 e^{-\ell_{*} / 2} \sinh t=2 i \sqrt{2 E},
$$

where $t$ is the saddle of $t_{1,2}$ and can be thought of as half of the (Lorentzian) Rindler angle spanned by the geodesic. The solutions of the saddle point equations are:

$$
\ell_{*}=\log \frac{16}{m^{2}+8 E}, \quad t=i \arctan \left(\frac{2 \sqrt{2 E}}{m}\right) .
$$




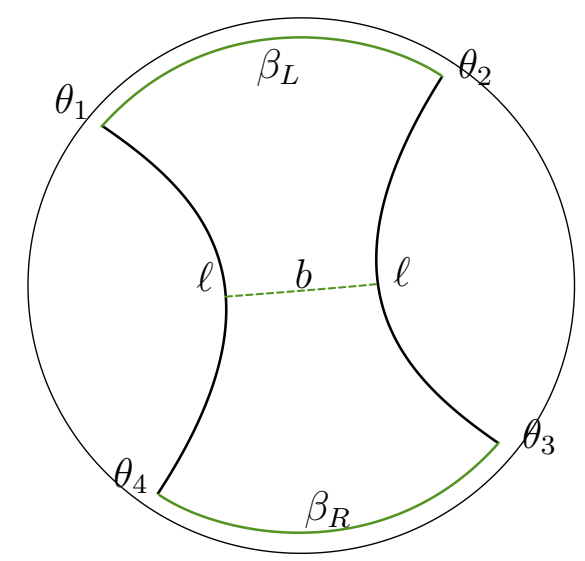

Figure 6: Construction of the wormhole geometry on the Poincaré disk.

Given $\ell_{*}, E$ and the length of the two asymptotic boundaries, the full geometry is specified. To fully characterize the geometry, it will be convenient to draw the wormhole geometry on the hyperbolic disk (figure 6) where we put the center of the two asymptotic boundaries at the center of the hyperbolic disk. In the Rindler coordinates $d s^{2}=d \rho^{2}+\sinh ^{2} \rho d \theta^{2}$, that is the location at $\rho=0$.

Since the four images of the operators will all at the same radius, we can denote their location as $\left(\rho_{b}, \theta_{1}\right)$ to $\left(\rho_{b}, \theta_{4}\right)$, where $\rho_{b}$ is purely determined by the energy $E$ and the IR cutoff $\epsilon$ :

$$
\sinh \rho_{b}=\frac{\beta_{E}}{2 \pi \epsilon}=\frac{1}{\sqrt{2 E} \epsilon} .
$$

The Rindler angle spanned by each of the asymptotic boundaries is also determined:

$$
\theta_{12,34}=\frac{2 \pi \beta_{L, R}}{\beta_{E}}=\sqrt{2 E} \beta_{L, R}
$$

The (regularized) geodesic distance between two bulk points $\left(\rho_{b}, \theta_{2}\right)$ and $\left(\rho_{b}, \theta_{3}\right)$ can be easily calculated by taking the inner product of their embedding coordinates, which gives:

$$
e^{\ell}=\epsilon^{2} e^{2 \rho_{b}} \sin ^{2}\left(\frac{\theta_{23}}{2}\right)=\frac{2}{E} \sin ^{2}\left(\frac{\theta_{23}}{2}\right)
$$

Compared with the second equation for the saddle point A.10, we confirmed the statement that $t$ is half of the Lorentzian Rindler angle spanned by the geodesic, $t=i \frac{\theta_{23}}{2}$. An important quantity in this geometry is the length of the geodesic across the wormhole $b$ which characterize the size of the wormhole. Due to the $\mathrm{SL}(2, \mathrm{R})$ invariance, $b$ is a function of the 
cross ratio of the four $\theta$ 's, and is therefore fully determined:

$$
\sinh \frac{b}{2}=\sqrt{\frac{\sin \frac{\theta_{12}}{2} \sin \frac{\theta_{34}}{2}}{\sin \frac{\theta_{32}}{2} \sin \frac{\theta_{42}}{2}}}=\frac{\sqrt{\sin \frac{\pi \beta_{L}}{\beta_{E}} \sin \frac{\pi \beta_{R}}{\beta_{E}}}}{|\sinh t|} .
$$

Then equation A.15 tells us that the size of the wormhole grows linearly with time for $\beta_{L, R} \sim \pm i T:$

$$
b \sim \frac{2 \pi T}{\beta_{E}}+2 \log \left(\left(\frac{m \beta_{E}}{4 \pi}\right)^{2}+1\right)
$$

\section{B State reconstruction in the canonical ensemble}

In this appendix, we discuss the canonical ensemble version of the state reconstruction studied in section 4. In the case of the microcanonical ensemble, we see that the $K$ charge states span the whole Hilbert space once $K$ exceeds the number of states in the energy window, i.e. after the Page transition.

The canonical ensemble can be understood as a distribution of the microcanonical ensemble weighted by the Boltzmann factor, and we can separate the states into Pre-Page states and Post-Page states. This suggests that for fixed $K$, the charge states can span the subspace of all the Post-Page states. As we increase $K$, this subspace will become larger as more and more states will hit the Page transition. Once $K \gg e^{2 S_{\mathrm{BH}}}$, most of the states that dominate the thermal distribution will become Post-Page and so any low energy excitation will become reconstructable by the $K$ charge states.

We can make this picture more precise using the Schwinger-Dyson (SD) equation (4.17). In the SD equation, we did not write down the explicit expression of $\rho(E)$ and $y\left(E_{1}, E_{2}\right)$. In the limit of small $G_{N}$, they has the following approximation:

$$
\rho(E) \sim e^{2 \pi \sqrt{2 E}} ; \quad y\left(E_{1}, E_{2}\right) \sim e^{-S_{0}-2 \pi \max \left(\sqrt{2 E_{1}}, \sqrt{2 E_{2}}\right)-\frac{\beta}{2}\left(E_{1}+E_{2}\right)} .
$$

The precise form of these two functions is not that important; rather, we should just observe that $\rho(E)$ is a density of states that is increasing as a function of energy, and $y\left(E_{1}, E_{2}\right)$ is a combination of the Boltzmann factor with the matrix element of the operator and decreases with the energy. This means that for fixed value of $R$, the integrand inside the SD equation (4.17) can be approximated as two functions according to the energy:

$$
\frac{y R}{1-y R} \sim \begin{cases}-1 ; & \left|y\left(E_{1}, E_{2}\right) R\right|>1 \\ y R ; & \left|y\left(E_{1}, E_{2}\right) R\right|<1\end{cases}
$$


The contour that separates these two regions is determined by equation $\left|y\left(E_{1}, E_{2}\right) R\right|=1$. Roughly speaking, this is saying that states that have energy $\left|y\left(E_{1}, E_{2}\right) R\right|<1$ can be well approximated by just the cylinder geometry and states have energy $\left|y\left(E_{1}, E_{2}\right) R>1\right|$ are well approximated by the pinwheel geometry. Typically, $R$ has only one square root branch cut in the complex $\lambda$ plane and at infinite $\lambda$ it asymptotes to $\frac{K}{\lambda}$. This means that near the lower end $\lambda_{0}$ of the branch cut, $R$ is large and negative. So we can use the approximation to simplify the SD equation near $\lambda_{0}$ :

$$
\lambda \sim \frac{K}{R}-\frac{1}{R} \int_{-y\left(E_{1}, E_{2}\right) R>1} d E_{1,2} \rho_{1,2}+\int_{-y\left(E_{1}, E_{2}\right) R<1} d E_{1,2} \rho_{1,2} y,
$$

where we use the notation $d E_{1,2} \rho_{1,2}$ to represent $d E_{1} d E_{2} e^{2 S_{0}} \rho\left(E_{1}\right) \rho\left(E_{2}\right)$. The branch point of the resolvent can therfore be determined by solving the equation $\frac{d \lambda}{d R}=0$. It is easy to see that the change of the integral domain cancels between the two integrals in equation (B.3), and we only left with equation:

$$
K=\int_{-y\left(E_{1}, E_{2}\right) R\left(\lambda_{0}\right)>1} d E_{1,2} \rho_{1,2} ; \quad \lambda_{0}=\int_{-y\left(E_{1}, E_{2}\right) R\left(\lambda_{0}\right)<1} d E_{1,2} \rho_{1,2} y
$$

The first equation determines the value of $R$ at $\lambda_{0}$.

The corresponding contour separates two regions in the energy plane, corresponding to the separation of Post-Page states (states that have energy $-y\left(E_{1}, E_{2}\right) R\left(\lambda_{0}\right)>1$ ) and PrePage states (states that have energy $-y\left(E_{1}, E_{2}\right) R\left(\lambda_{0}\right)<1$ ). The second equation then tells us that all the Pre-Page states contribute together as a shift of the end point of the branchcut, away from zero. For $\lambda>\lambda_{0}$, the magnitude of $R$ will be smaller than $R\left(\lambda_{0}\right)$. This means that the integral in the original SD equation 4.17) can be separated into two regions based on whether the energies are Pre-Page or Post-Page:

$$
\lambda R \sim K+\int_{-y\left(E_{1}, E_{2}\right) R\left(\lambda_{0}\right)>1} d E_{1,2} \rho_{1,2} \frac{y R}{1-y R}+\lambda_{0} R .
$$

which in the limit of large $K$ and $\lambda$ away from $\lambda_{0}$, it has approximate solution:

$$
R \sim \frac{K}{\lambda-\lambda_{0}}
$$

Plugging this in equation : $V M^{-1} V$ : and deforming the integration contour of $\lambda$ around $\lambda_{0}$, we find:

$$
: V M^{-1} V:=\int d E_{1,2} \rho_{1,2} y \oint \frac{d \lambda}{2 \pi i \lambda} \frac{y R}{1-y R} \sim \int d E_{1,2} \rho_{1,2} y \frac{y K}{\lambda_{0}+y K}
$$

Once again, this separate the energy integral into two regions depends on the relative value between $\lambda_{0}$ and $y K$ : for the energy range that $y K>\lambda_{0}$, we have contribution 
$\int_{y K>\lambda_{0}} d E_{1,2} \rho_{1,2} y$ which reconstructs the energy component of state $|\psi\rangle$ in this region; for the energy range that $y K<\lambda_{0}$, we have contribution $\frac{K}{\lambda_{0}} \int_{y K>\lambda_{0}} d E_{1,2} \rho_{1,2} y^{2}$ which quickly decays to zero when $K>e^{2 S_{\mathrm{BH}}}$. As a consequence, this means that the fixed $K>e^{2 S_{\mathrm{BH}}}$ charge states span a complete basis in the states whose energy satisfies the following condition:

$$
y\left(E_{1}, E_{2}\right)>\frac{\lambda_{0}}{K} .
$$

\section{References}

[1] C. W. Misner and J. A. Wheeler, "Classical physics as geometry: Gravitation, electromagnetism, unquantized charge, and mass as properties of curved empty space," Annals Phys. 2 (1957) 525-603.

[2] S. B. Giddings and A. Strominger, "Loss of Incoherence and Determination of Coupling Constants in Quantum Gravity," Nucl. Phys. B 307 (1988) 854-866.

[3] R. Kallosh, A. D. Linde, D. A. Linde, and L. Susskind, "Gravity and global symmetries," Phys. Rev. D 52 (1995) 912-935, arXiv:hep-th/9502069.

[4] J. Polchinski, "Monopoles, duality, and string theory," Int. J. Mod. Phys. A 19S1 (2004) 145-156, arXiv: hep-th/0304042.

[5] N. Arkani-Hamed, L. Motl, A. Nicolis, and C. Vafa, "The String landscape, black holes and gravity as the weakest force," JHEP 06 (2007) 060, arXiv: hep-th/0601001.

[6] T. Banks and N. Seiberg, "Symmetries and Strings in Field Theory and Gravity," Phys. Rev. D 83 (2011) 084019, arXiv:1011.5120 [hep-th].

[7] D. Harlow and H. Ooguri, "Constraints on Symmetries from Holography," Phys. Rev. Lett. 122 no. 19, (2019) 191601, arXiv:1810.05337 [hep-th]

[8] D. Harlow and H. Ooguri, "Symmetries in quantum field theory and quantum gravity," arXiv:1810.05338 [hep-th]

[9] D. Harlow and E. Shaghoulian, "Global symmetry, Euclidean gravity, and the black hole information problem," arXiv:2010.10539 [hep-th].

[10] S. W. HAWKING, "Black hole explosions?," Nature 248 no. 5443, (Mar, 1974) 30-31. https://doi.org/10.1038/248030a0.

[11] S. W. Hawking, "Particle creation by black holes," Communications in Mathematical Physics 43 no. 3, (Aug, 1975) 199-220, https://doi.org/10.1007/BF02345020. 
[12] S. Hawking, "The Unpredictability of Quantum Gravity," Commun. Math. Phys. 87 (1982) 395-415.

[13] A. Almheiri, T. Hartman, J. Maldacena, E. Shaghoulian, and A. Tajdini, "The entropy of Hawking radiation," arXiv:2006.06872 [hep-th].

[14] L. Abbott and M. B. Wise, "Wormholes and global symmetries," Nuclear Physics B 325 no. 3, (1989) $687-704$. http://www.sciencedirect.com/science/article/pii/0550321389905038.

[15] G. Penington, S. H. Shenker, D. Stanford, and Z. Yang, "Replica wormholes and the black hole interior," arXiv:1911.11977 [hep-th].

[16] A. Almheiri, T. Hartman, J. Maldacena, E. Shaghoulian, and A. Tajdini, "Replica Wormholes and the Entropy of Hawking Radiation," JHEP 05 (2020) 013 , arXiv:1911.12333 [hep-th].

[17] J. M. Maldacena and L. Maoz, "Wormholes in AdS," JHEP 02 (2004) 053, arXiv:hep-th/0401024.

[18] E. Witten, "Anti-de Sitter space and holography," Adv. Theor. Math. Phys. 2 (1998) 253-291, arXiv:hep-th/9802150.

[19] S. Sachdev, "Bekenstein-Hawking Entropy and Strange Metals," Phys. Rev. X 5 no. 4, (2015) 041025, arXiv:1506.05111 [hep-th].

[20] A. Kitaev, "Talks given at the Fundamental Physics Prize Symposium and KITP seminars,".

[21] J. Maldacena and D. Stanford, "Remarks on the Sachdev-Ye-Kitaev model," Phys. Rev. D94 no. 10, (2016) 106002, arXiv:1604.07818 [hep-th].

[22] A. Kitaev and S. J. Suh, "The soft mode in the Sachdev-Ye-Kitaev model and its gravity dual," JHEP 05 (2018) 183, arXiv:1711.08467 [hep-th].

[23] C. Teitelboim, "Gravitation and Hamiltonian Structure in Two Space-Time Dimensions," Phys. Lett. B126 (1983) 41-45.

[24] R. Jackiw, "Lower Dimensional Gravity," Nucl. Phys. B252 (1985) 343-356.

[25] Y. Chen and H. W. Lin, "Signatures of global symmetry violation in relative entropies and replica wormholes," arXiv:2011.06005 [hep-th]. 
[26] W. Israel, "Event horizons in static vacuum space-times," Phys. Rev. 164 (1967) $1776-1779$.

[27] W. Israel, "Event horizons in static electrovac space-times," Commun. Math. Phys. 8 (1968) 245-260.

[28] B. Carter, "Axisymmetric Black Hole Has Only Two Degrees of Freedom," Phys. Rev. Lett. 26 (1971) 331-333.

[29] D. Robinson, "Uniqueness of the Kerr black hole," Phys. Rev. Lett. 34 (1975) 905-906.

[30] R. Bousso, "A Covariant entropy conjecture," JHEP 07 (1999) 004, arXiv:hep-th/9905177.

[31] R. Bousso, H. Casini, Z. Fisher, and J. Maldacena, "Proof of a Quantum Bousso Bound," Phys. Rev. D 90 no. 4, (2014) 044002, arXiv:1404.5635 [hep-th].

[32] S. B. Giddings, "Constraints on black hole remnants," Phys. Rev. D 49 (1994) 947-957, arXiv:hep-th/9304027.

[33] L. Susskind, "Trouble for remnants," arXiv:hep-th/9501106.

[34] P. Saad, "Late Time Correlation Functions, Baby Universes, and ETH in JT Gravity," arXiv:1910.10311 [hep-th].

[35] G. Penington, "Entanglement Wedge Reconstruction and the Information Paradox," JHEP 09 (2020) 002, arXiv: 1905.08255 [hep-th].

[36] A. Almheiri, N. Engelhardt, D. Marolf, and H. Maxfield, "The entropy of bulk quantum fields and the entanglement wedge of an evaporating black hole," JHEP 12 (2019) 063, arXiv:1905.08762 [hep-th].

[37] A. Almheiri, R. Mahajan, J. Maldacena, and Y. Zhao, "The Page curve of Hawking radiation from semiclassical geometry," JHEP 03 (2020) 149, arXiv:1908.10996 [hep-th].

[38] N. Engelhardt and A. C. Wall, "Quantum Extremal Surfaces: Holographic Entanglement Entropy beyond the Classical Regime," JHEP 01 (2015) 073, arXiv:1408.3203 [hep-th].

[39] P. Saad, S. H. Shenker, and D. Stanford, "JT gravity as a matrix integral," arXiv:1903.11115 [hep-th].

[40] D. Stanford, "More quantum noise from wormholes," arXiv:2008.08570 [hep-th]. 
[41] T. G. Mertens, G. J. Turiaci, and H. L. Verlinde, "Solving the Schwarzian via the Conformal Bootstrap," JHEP 08 (2017) 136, arXiv:1705.08408 [hep-th].

[42] A. Kitaev and S. J. Suh, "Statistical mechanics of a two-dimensional black hole," JHEP 05 (2019) 198, arXiv: 1808.07032 [hep-th]

[43] Z. Yang, "The Quantum Gravity Dynamics of Near Extremal Black Holes," JHEP 05 (2019) 205, arXiv: 1809.08647 [hep-th]

[44] A. Blommaert, T. G. Mertens, and H. Verschelde, "The Schwarzian Theory - A Wilson Line Perspective," JHEP 12 (2018) 022, arXiv:1806.07765 [hep-th].

[45] L. V. Iliesiu, S. S. Pufu, H. Verlinde, and Y. Wang, "An exact quantization of Jackiw-Teitelboim gravity," JHEP 11 (2019) 091, arXiv: 1905.02726 [hep-th].

[46] E. Witten, "On quantum gauge theories in two-dimensions," Commun. Math. Phys. 141 (1991) 153-209.

[47] E. Witten, "Two-dimensional gauge theories revisited," J. Geom. Phys. 9 (1992) 303-368, arXiv:hep-th/9204083.

[48] L. V. Iliesiu, "On 2D gauge theories in Jackiw-Teitelboim gravity," arXiv:1909.05253 [hep-th].

[49] D. Kapec, R. Mahajan, and D. Stanford, "Matrix ensembles with global symmetries and 't Hooft anomalies from 2d gauge theory," JHEP 04 (2020) 186, arXiv:1912.12285 [hep-th].

[50] H. Maxfield and G. J. Turiaci, "The path integral of 3D gravity near extremality; or, JT gravity with defects as a matrix integral," arXiv:2006.11317 [hep-th].

[51] E. Witten, "Matrix Models and Deformations of JT Gravity," arXiv:2006.13414 [hep-th].

[52] J. M. Maldacena, G. W. Moore, and N. Seiberg, "D-brane charges in five-brane backgrounds," JHEP 10 (2001) 005, arXiv: hep-th/0108152.

[53] A. Kapustin and N. Seiberg, "Coupling a QFT to a TQFT and Duality," JHEP 04 (2014) 001, arXiv:1401.0740 [hep-th].

[54] D. Gaiotto, A. Kapustin, N. Seiberg, and B. Willett, "Generalized Global Symmetries," JHEP 02 (2015) 172, arXiv:1412.5148 [hep-th]. 
[55] D. Stanford and E. Witten, "JT Gravity and the Ensembles of Random Matrix Theory," arXiv:1907.03363 [hep-th].

[56] P. Saad, S. H. Shenker, and D. Stanford, "A semiclassical ramp in SYK and in gravity," arXiv:1806.06840 [hep-th].

[57] D. Marolf and H. Maxfield, "Transcending the ensemble: baby universes, spacetime wormholes, and the order and disorder of black hole information," JHEP 08 (2020) 044, arXiv: 2002.08950 [hep-th].

[58] X. Dong and H. Wang, "Enhanced corrections near holographic entanglement transitions: a chaotic case study," JHEP 11 (2020) 007, arXiv:2006.10051 [hep-th]

[59] D. Marolf, S. Wang, and Z. Wang, "Probing phase transitions of holographic entanglement entropy with fixed area states," arXiv:2006.10089 [hep-th]

[60] C. Akers and G. Penington, "Leading order corrections to the quantum extremal surface prescription," arXiv:2008.03319 [hep-th].

[61] L. V. Iliesiu and G. J. Turiaci, "The statistical mechanics of near-extremal black holes," arXiv:2003.02860 [hep-th]

[62] P. Nayak, A. Shukla, R. M. Soni, S. P. Trivedi, and V. Vishal, "On the Dynamics of Near-Extremal Black Holes," JHEP 09 (2018) 048, arXiv:1802.09547 [hep-th].

[63] U. Moitra, S. P. Trivedi, and V. Vishal, "Extremal and near-extremal black holes and near-CFT $1, "$ JHEP 07 (2019) 055, arXiv: 1808.08239 [hep-th]. 